\title{
Tailoring the Morphology and the Optical Properties of Semiconductor Nanocrystals by Alloying
}

\author{
Young-Kuk Kim and Chul-Jin Choi \\ Korea Institute of Materials Science \\ Republic of Korea
}

\section{Introduction}

Colloidal semiconductor nanocrystals (NCs) or quantum dots (QDs) based on semiconductors open possibilities for applications in new areas (Bawendi et al., 1990; Alivisatos, 1996; Markovich et al., 1999). Since hot injection synthesis of mono-dispersed spherical nanocrystals using organometallic precursors was introduced by an MIT group (Murray et al., 1993), extensive works have been devoted to make variation of their size and shape, compositions. In this chapter, we will focus on the effect of the alloying or impurity doping on the shape and the optical properties of the NCs.

In the first section, we will introduce and discuss the effect of alloying on the morphology of semiconductor NCs. The effects of $\mathrm{Pb}^{2+}$ addition on the morphological development of CdSe nanocrystals was discussed. The addition of $\mathrm{Pb}$ ions in the initial precursor solution for the synthesis of alloyed CdSe NCs changed the morphology of CdSe nanocrystals to branched rod with high aspect ratio. The branched nanocrystals are mainly composed of wurzite phase grown along [001] direction and the length of rods in each branched nanocrystal can be increased by increasing amount of $\mathrm{Pb}^{2+}$ addition to accelerate anisotropic growth of nanocrystals. The luminescence, however, mostly arises from trap-related recombination and significantly red-shifted by $\mathrm{Pb}^{2+}$ addition. Moreover, it will be shown that the zinc blend-wurzite polymorphism was popularly observed in these branched CdSe NCs and the twinned structure gave rise to highly asymmetric line shape of photoluminescence spectra. Based on the known optical properties, we will further discuss the application of the branched CdSe NCs to the photovoltaic devices.

In the second section, we discussed the effect of the alloying on the optical properties of Cdfree I-III-VI 2 group semiconductor nanocrystals. Although, many works have been focused on II-VI semiconductors such as CdSe, CdS, CdTe, etc. due to their high quantum yield of fluorescence with tunable emission wavelength, incorporation of toxic heavy metals such as $\mathrm{Cd}, \mathrm{Pb}, \mathrm{Hg}$ in the II-VI semiconductors have limited their public application. As alternatives to II-VI semiconductors, several materials system including III-V semiconductor NCs (e.g. $\mathrm{InP}$ ) and impurity-doped ZnS NCs have been developed. However, each material system have some drawbacks such as requirement of expensive pyrophoric compounds as precursors, chemical stability, tunability of emission wavelength, etc. Ternary chalcopyritetype I-III-VI $\mathrm{V}_{2}$ group semiconductor NCs were also synthesized as less-toxic new candidates 
to II-VI semiconductor NCs. I-III-VI 2 group semiconductors with appropriate band-gap energies (e.g. $1.5 \mathrm{eV}$ for $\mathrm{CuInS}_{2}$ and $1.68 \mathrm{eV}$ for $\mathrm{CuGaSe}_{2}$ ) are direct transition semiconductors favoring ionic bonding similar to II-VI semiconductors. However, in contrast to II-VI group semiconductors which make luminescence by direct exciton recombination, the dominant luminescence mechanism for the chalcopyrite-type I-III-VI 2 group semiconductor NCs in the visible spectrum is known to be "donor-acceptor" pair recombination (DAP) which requires defects to play an important role in the luminescence process. Recently, several results have been reported for synthesis of non-aggregated I-III-VI 2 group semiconductor NCs of a few nanometers with fluorescence quantum yield of a few percent. We discussed the luminescence mechanism of those nanocrystals and delineate the effect of alloying on their luminescence. The photoluminescence of ZnS alloyed NCs show large blue-shift with respect to pure I-III-VI 2 NCs. It was shown that the emission wavelength and intensities of IIII-VI $2 \mathrm{NCs}$ can be tuned by alloying. In addition, we intended to report a one-pot synthesis route to highly luminescent I-III-VI - -based core-shell NCs with high quantum yield through defect control and shell formation by refluxing with zinc acetate and palmitic acid. Zinc acetate played an important role in formation of inorganic shell and size regularization of IIII-VI 2 NCs. We showed that Cu-deficiency in precursor solution is essential to enhancing quantum yield and surface modification by refluxing with zinc acetate and palmitic acid enables a reproducible synthesis of monodispersed highly luminescent I-III-VI $\mathrm{NCs}$ with less toxic and highly stable precursors.

\section{Tailoring the morphology of CdSe nanocrystals by alloying with $\mathrm{Pb}$}

The size and shape of semiconductor nanocrystals play an important role to determine their electronic and optic properties. In particular, anisotropic nanocrystal exhibit unique performance such as enhanced solar cell efficiency, polarized luminescence, etc. Thus, anisotropic nanocrystals with variety of compositions such as CdS, CdTe, CdSe, etc. were synthesized with hot injection method (Manna et al., 2000; Zhang \& Yu, 2006; Li \& Wang, 2003). In particular, tetrapod-shaped CdTe nanocrystals were synthesized with high shape selectivity and high size uniformity (Zhang \& Yu, 2006). In contrast to this, CdSe nanocrystals were relatively hard to synthesize with high shape selectivity, since the energy difference between the wurzite and the zinc blende is too small to facilitate tetrapod-shaped crystals (Zhang \& Yu, 2006; Asokan et al., 2007). Recently, Asokan et al. reported synthesis of CdSe tetrapods with high shape selectivity and high size uniformity (Asokan et al., 2007). Most of syntheses of anisotropic CdSe nanocrystals involve special surfactants such as alkylphosphonic acid, quaternary ammonium salt compounds, etc. These surfactant ligands preferentially bind to certain particle facets to reduce growth rate of these facets.

In addition, doping in semiconductor nanocrystals can adjust their optical and electronic properties (Erwin, 2005). For example, doping foreign atoms can introduce characteristic luminescence peaks in photoluminescence spectra of nanocrystals and reduce the lasing threshold of semiconductor nanocrystals. Thus, works on doped nanocrystals are mainly devoted to tune the optical and electronic properties of nanocrystals. To our knowledge, reports on the morphological development of nanocrystal by doping are quite rare (Hsu \& $\mathrm{Lu}, 2008)$. In this study, we report the effect of $\mathrm{Pb}^{2+}$ addition on the morphological development of CdSe nanocrystals and their structure-property relationships were analyzed. 


\subsection{Synthesis of $(\mathrm{Cd}, \mathrm{Pb})$ Se alloyed nanocrystals}

CdSe nanocrystals were synthesized by injection of selenium precursor solution into hot $\mathrm{Cd}$ precursor solution based on the synthesis method for spherical CdSe nanocrystals (Asokan et al., 2005). The $\mathrm{Cd}$ precursor solution was synthesized by dissolving $4 \mathrm{mmol} \mathrm{CdO}$ to the mixture of $10 \mathrm{mmol}$ oleic acid and $20 \mathrm{ml}$ 1-octadecene (ODE). Sometimes, lead (II) acetate hydrate was intentionally added instead of the same amount of $\mathrm{CdO}$ maintaining total concentration of metal salts. Subsequently, the mixtures were heated to $150^{\circ} \mathrm{C}$ under $\mathrm{N}_{2}$ gas flow and metallic precursors were completely dissolved to form clear solution of metal oleate (Solution A). Separately, $8 \mathrm{mmol}$ selenium powders were dissolved in $7.71 \mathrm{ml}$ trioctylphosphine (TOP) under dry $\mathrm{N}_{2}$ atmosphere in the glove box and the selenium solution was swiftly injected to $\mathrm{Cd}$-based precursor solution A at $225^{\circ} \mathrm{C}$. Aliquots were taken at different time interval after injection and rapidly cooled to room temperature. Before further characterization, acetone was added to precipitate CdSe-based nanocrystals and the precipitates were purified by repetition of centrifugation and re-dispersion to toluene. Overall reaction occurred in this synthesis reaction can be written as $(1-x)\left[C d(A)_{n 1}\right]$ $+\mathrm{x}\left[\mathrm{Pb}(\mathrm{A})_{\mathrm{n} 1}\right]+\mathrm{Se}(\mathrm{B})_{\mathrm{n} 2} \Rightarrow \mathrm{Cd}_{1-\mathrm{x}} \mathrm{Pb}_{\mathrm{x}} \mathrm{Se}+\mathrm{n}_{1} \mathrm{~A}+\mathrm{n}_{2} \mathrm{~B}$, where $\mathrm{A}$ and $\mathrm{B}$ corresponds to oleic acid and trioctylphosphine, respectively.

\subsection{Effect of $\mathrm{Pb}$ addition on the morphology of $\mathrm{CdSe}$ nanocrystals}

The crystal structure and phase formation of nanocrystals were characterized by powder Xray diffraction (XRD) taken using RINT2000 (Rigaku, Japan) with $\mathrm{Cu}-K \alpha$ line as the X-ray source. Fig. 2.1(a) shows XRD patterns of nanocrystals grown at $225^{\circ} \mathrm{C}$ for 300 seconds with various initial abundance of $\mathrm{Pb}^{2+}$ in the precursor solution. Irrespective of their initial abundance of $\mathrm{Pb}$ in the solution, XRD patterns of nanocrystals are consistent with that of hexagonal wurzite $\mathrm{CdSe}$ and displaying no trace of impurity peaks. The more $\mathrm{Pb}^{2+}$ ions were contained in the initial precursor solution, the narrower peaks in the XRD patterns became. Uniquely, the width of (002) peaks in the XRD pattern of CdSe nanocrystals was drastically decreased with increasing initial $\mathrm{Pb}^{2+}$ content in the reaction mixtures. The sharp (002) peak in the XRD pattern implies a preferential growth of CdSe nanocrystals along [001] direction. This preferential growth of CdSe nanocrystals resulted in highly anisotropic shape such as rod, tetrapod, branched crystals, etc. Furthermore, the narrower (002) peak with increasing $\mathrm{Pb}$ content in the reaction mixture indicates that longer nanocrystals were synthesized from the precursor solution containing more $\mathrm{Pb}^{2+}$.

The high resolution TEM (HR-TEM) image of Pb-containing nanocrystals presented in Fig. 2.2(a) showed highly entangled and branched morphology. Analysis of the lattice image showed that the planar spacing along axis of nanorods is about $0.35 \mathrm{~nm}$, which corresponds to planar spacing of (002) planes in the wurzite CdSe. Again, it was confirmed that CdSe crystals were preferentially grown along [002] direction. The junction point of the two branches is composed of zinc blende phase as indicated by dotted circle in Fig. 2.2(a).

Fig. 2.2(b) showed the abundance of $\mathrm{Pb}$ in the grown nanocrystals, $\mathrm{Pb} /(\mathrm{Cd}+\mathrm{Pb})$ measured by induction coupled plasma-mass spectroscopy (ICP-MS). Pb content increased linearly with the initial amount of $\mathrm{Pb}$ in the precursor solution. Although $\mathrm{Pb}^{2+}$ can be completely soluble in CdSe within 40\% (Hankare et al., 2005), the solubility of Pb in CdSe nanocrystals is highly limited due to the fast kinetics of nanocrystal growth at $225^{\circ} \mathrm{C}$. The $\mathrm{Pb}$ content in the CdSe nanocrystals is decreased with increasing reaction time at $225^{\circ} \mathrm{C}$ as shown in Fig. 2(b). That is, a relatively large amount of $\mathrm{Pb}$ is incorporated in nanocrystals just after Se 
injection at $225^{\circ} \mathrm{C}$ and smaller amount of $\mathrm{Pb}$ is incorporated in the nanocrystals during the growth of each branch.
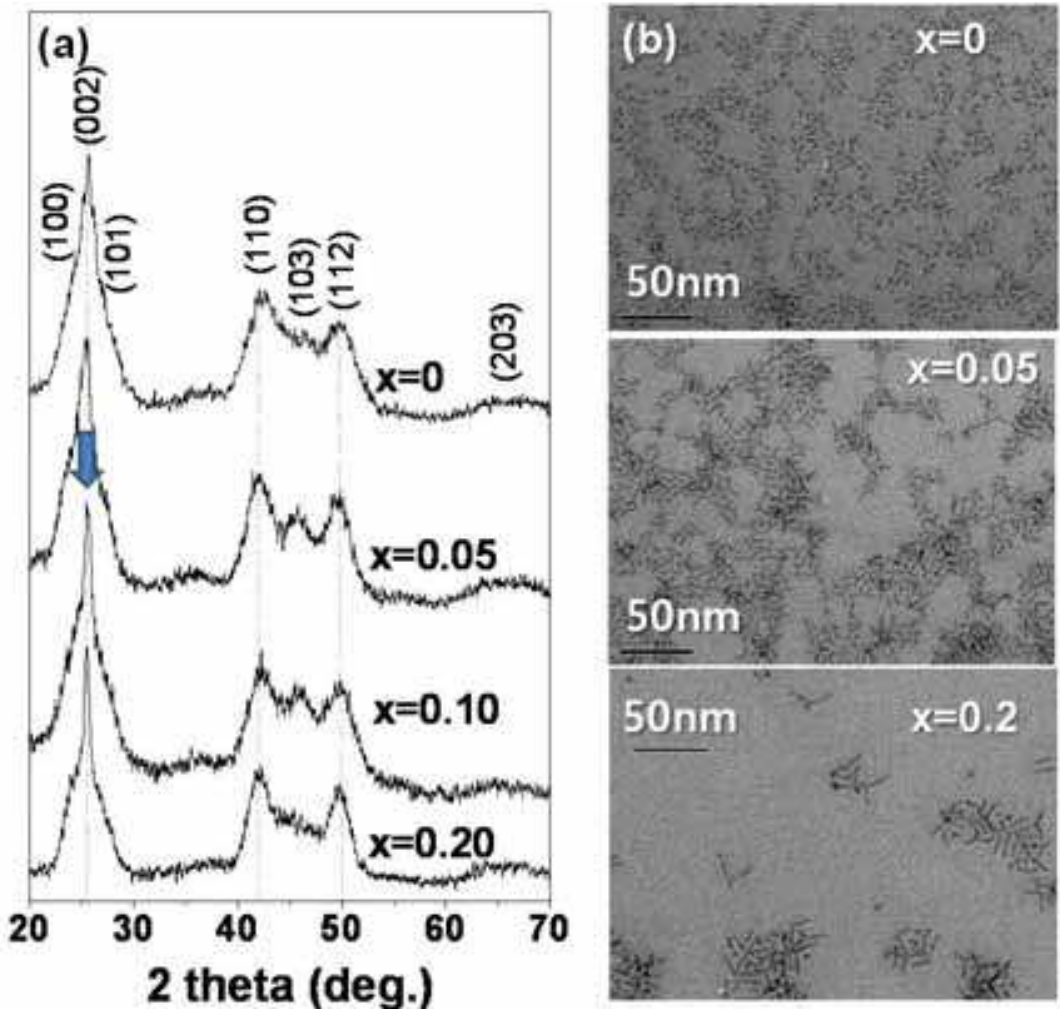

Fig. 2.1. (a) X-ray diffraction (XRD) patterns and TEM images of nanocrystals grown at $225^{\circ} \mathrm{C}$ with various initial molar abundance of $\mathrm{Pb}, \mathrm{x}=\mathrm{Pb} /(\mathrm{Cd}+\mathrm{Pb})$. Peak indices in the XRD pattern were assigned according to wurzite CdSe.

(a)

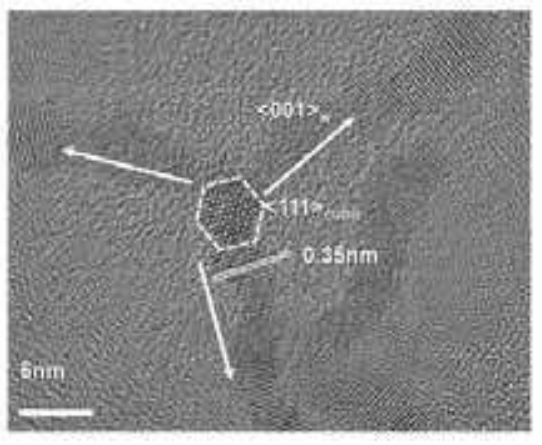

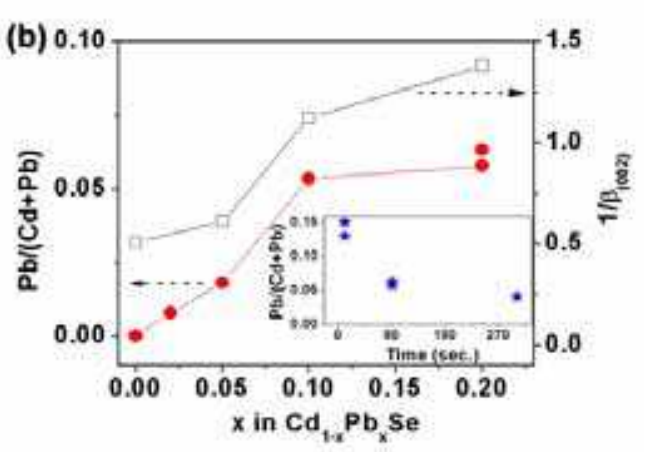

Fig. 2.2. (a) High resolution TEM images for nanocrystals grown from $\mathrm{Cd}_{0.8} \mathrm{~Pb}_{0.2} \mathrm{Se}$ precursor solution and $(\mathrm{b})$ the abundance of $\mathrm{Pb}$ in the grown nanocrystals, $\mathrm{Pb} /(\mathrm{Cd}+\mathrm{Pb})$. 
Since the reciprocal value of FWHM ( $\beta$ ) for (002) peaks in the XRD patterns is proportional to length (L) of each branch in the nanocrystals according to Scherrer's formula $(\mathrm{L}=0.94 \lambda / \beta \cos \theta)$, the monotonous increase in $1 / \beta_{(002)}$ indicates linear increase in the length of branches of the nanocrystals by $\mathrm{Pb}$ addition. Thus, the initial abundance of $\mathrm{Pb}$ controls the morphological development of nanocrystals. Raman spectroscopy has been used to study the structure of nanocrystals. Specimen for Raman spectra were prepared by dropping solution containing nanocrystals onto silicon wafer. After drying, $\mathrm{Ar}^{+}$laser $(\lambda=514.5 \mathrm{~nm}$, power less than $10 \mathrm{~mW}$ ) was focused on a spot of $50 \mu \mathrm{m}$ diameter in each sample and Raman spectra were collected with a triple Raman spectrometer (SPEX-1430). Fig.2.4(a) showed a Raman spectrum of the nanocrystals grown at $225^{\circ} \mathrm{C}$. The first order Raman band was identified at $\omega=206 \mathrm{~cm}^{-1}$ for nanocrystals grown from Pb-free precursor solution for 90 seconds at $225^{\circ} \mathrm{C}$ and $\omega=207 \mathrm{~cm}^{-1}$ for nanocrystals grown from $\mathrm{Pb}$-containing precursor solution under the same growth condition. The observed Raman spectra are highly asymmetric and shifted from the value of bulk CdSe $\left(\omega=210 \mathrm{~cm}^{-1}\right)$. The asymmetric line shape of the Raman band is commonly observed in low dimensional nanostructures and can be fitted well with two Lorentzian peak functions which is explained with two effects. The more pronounced peak at $\omega=206 \mathrm{~cm}^{-1}$ can be assigned to be a CdSe longitudinal optical (LO) mode and the shoulder on the low frequency side of LO mode is related to surface optical phonon (SO) mode (Lange et al., 2009; Comas et al., 2002).
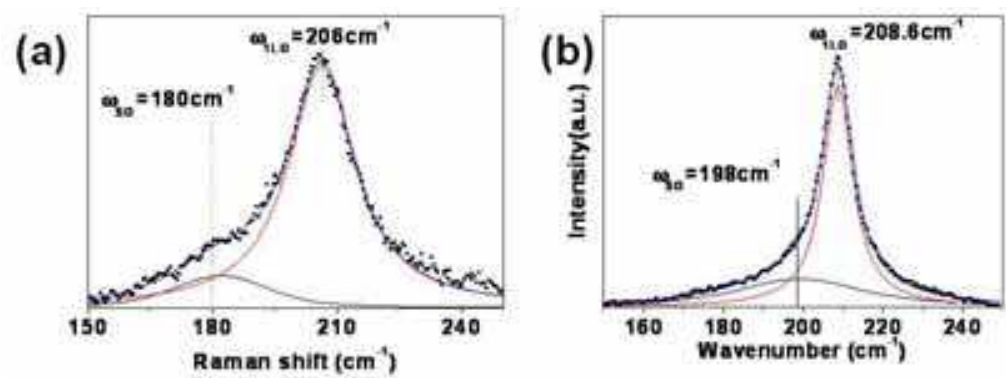

Fig. 2.3. Raman spectra of the nanocrystals grown at $225^{\circ} \mathrm{C}$ from (a) Pb-free precursor solution and (b) solution containing $\mathrm{Pb}$ with $\mathrm{x}=0.2$.

The SO mode can be observed in the Raman scattering process due to non-spherical geometry of nanocrystals and it is highly dependent on the aspect ratio of nanocrystals. As shown in Fig. 2.3(b), the frequency of SO mode for nanocrystals grown from $\mathrm{Pb}$-containing precursor solution shifted to high frequency. That is, the frequency of SO mode for nanocrystals grown from $\mathrm{Pb}$-free precursor solution is much lower than that for nanocrystals grown from $\mathrm{Pb}$-containing precursor solution at the same growth conditions. Lange et al. showed that Raman-active SO mode of CdSe nanorods is shifted to high frequency, as the aspect ratio (length/diameter) increases (Comas et al., 2002). Again, this indicated that the nanocrystals with high aspect ratio can be synthesized from $\mathrm{Pb}$-containing precursor solution.

We noted that the emission spectra of nanocrystals were symmetric and sharp, which indicates narrow size distribution of the grown nanocrystals. Most of emission spectra from the branched nanocrystals detected at the room temperature were from band-edge emission as shown in Fig. 2.4(a). However, they showed drastic reduction in their intensity with 
increasing amount of $\mathrm{Pb}$ in the precursor solution. In general, emission from semiconductor nanocrystals is composed of band-edge emission and emission from surface trap states (Saunders et al., 2008; Jing et al., 2009). Temperature-dependent PL spectroscopy is often used to study the radiative and the non-radiative relaxation process in nanocrystals. Contrast to the room temperature PL spectrum, the low temperature PL spectrum of the nanocrystals grown from $\mathrm{Pb}$-containing precursor solution exhibited two peaks: a narrow peak at $620 \mathrm{~nm}$ due to band-edge emission (BE) and a broad intense peak highly red-shifted from the band edge, centered at about $800 \mathrm{~nm}$, which results from trap-related emission (TE). In the low temperature PL spectrum, majority of the emission spectrum emanated from TE. The large surface-to-volume ratio of branched nanocrystals with high aspect ratio increased the occurrence of surface trap states and reduced the band-edge emission.
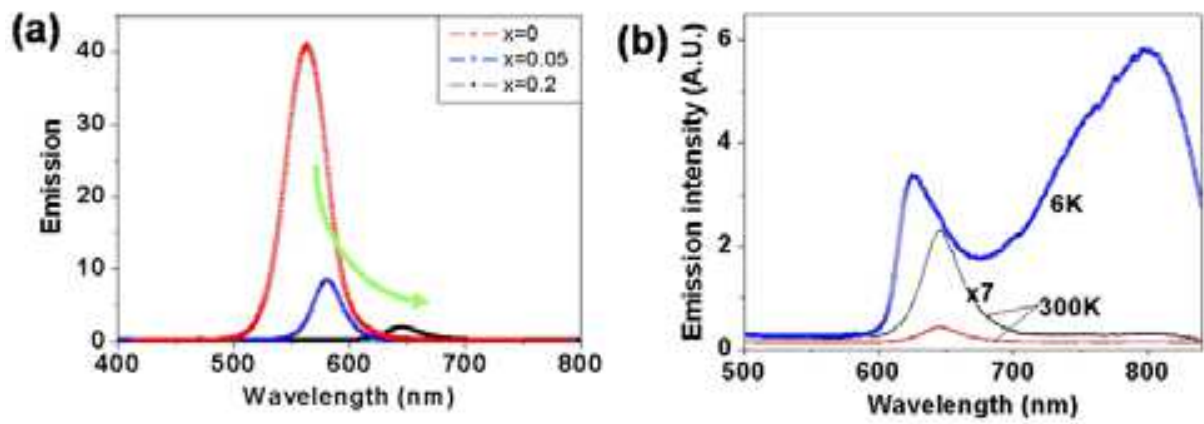

Fig. 2.4. (a) Room temperature photoluminescence of $\mathrm{Cd}_{1-\mathrm{x}} \mathrm{Pb}_{\mathrm{x}} \mathrm{Se}$ with various $\mathrm{Pb}$-content $(\mathrm{x})$ and $(b)$ temperature dependence of photoluminescence spectra for $\mathrm{Cd}_{1-x} \mathrm{~Pb}_{x} \mathrm{Se}(\mathrm{x}=0.2)$ nanocrystals grown at $225^{\circ} \mathrm{C}$.

Two mechanisms working on the formation of branched nanocrystals have been suggested (Kudera et al., 2008). The formation of branched nanocrystals was commonly based on the initial formation of octahedral crystallite and the subsequent growth of anisotropic crystallites from the facets of the octahedra. One model for the branched nanocrystal formation is the octa-twin model which was developed at the early 1990s for tetrapods of $\mathrm{ZnS}$ and $\mathrm{ZnO}$. This model describes the initial octahedral crystallites as cores composed of eight wurzite domains with tetrahedral shape. In this model, branches are grown on $\{0001\}$ facets of wurzite cores and form branched nanocrystals with multi-pods. The other mechanism suggested is the polymorphism model which describes the formation of branched nanocrystals as initial formation of octahedral cores with zinc blende structure and further growth of branches on the $\{111\}$ facets of cubic cores. Since the formation energy difference between cubic zinc blende structure and hexagonal wurzite structure for CdSe is quite small, co-existence of both phases in a crystal is not rare. In particular, during the synthesis of CdSe, injection of Se precursors can drop the temperature and zinc blende nuclei readily form by the temperature effect. In addition to temperature effect, the change in crystalline phase can be justified by the affinity of ligands to certain facets (Manna et al., 2000). Special ligands such as alkylphosphonic acid can tightly bind the facets of wurzite and induce the formation of wurzite crystallites.

For $\mathrm{Cd}_{1-\mathrm{x}} \mathrm{Pb}$ Se alloyed nanocrystals, those two mechanisms cannot explain the formation of branched nanocrystals without modification, since oleic acid was used as capping ligands 

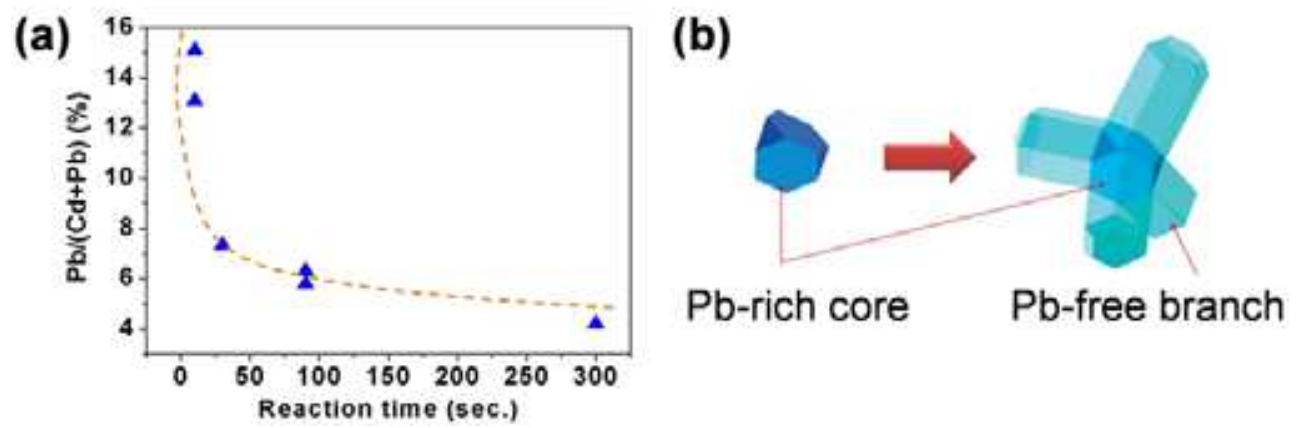

Fig. 2.5. (a) Variation of the incorporated $\mathrm{Pb}$ content with respect to reaction time and (b) a schematic illustration of core-branch growth in $\mathrm{Cd}_{1-x} \mathrm{~Pb}_{\mathrm{x}} \mathrm{Se}(\mathrm{x}=0.2)$ nanocrystals.

and nearly isotropic nanocrystals were synthesized in the absence of $\mathrm{Pb}$. Fig. 2.5(a) shows the variation of $\mathrm{Pb}$-content in the branched nanocrystals, $\mathrm{Cd}_{1-\mathrm{x}} \mathrm{Pb}_{\mathrm{x}} \mathrm{Se}(\mathrm{x}=0.2)$. At the initial nucleation-growth stage, the concentration of $\mathrm{Pb}$ was $c a .15 \%$ and rapidly decreased to be less than $8 \%$ after this stage. That is, a relatively large amount of $\mathrm{Pb}$ is incorporated in nanocrystals just after Se injection at $225{ }^{\circ} \mathrm{C}$ and the incorporation of $\mathrm{Pb}$ was highly suppressed during the growth of each branch. One possible explanation on the formation of branched CdSe nanocrystals by alloying with $\mathrm{Pb}$ is that nuclei with cubic zinc blende structure were formed by incorporation of $\mathrm{Pb}$ during the nucleation step and further growth wurzite branch on (111) facet of cubic crystallites as suggested in the polytypism model []. In particular, the low $\mathrm{Pb}$ content in the CdSe nanocrystals after prolonged reaction at $225^{\circ} \mathrm{C}$ can be explained by "self-purification effect" (Dalpian \& Chelikowsky, 2006), which assess migration of impurities or lattice defect to the surface of nanocrystals (Erwin et al, 2005). However, further study is now in progress to elucidate the formation mechanism of branched nanocrystals in $\mathrm{Cd}-\mathrm{Pb}-\mathrm{Se}$ system.

\section{Tailoring the optical properties of nanocrystals by alloying}

The semiconductor nanocrystals were characterized with strong emission of the visible light and their continuous tuning by size due to quantum confinement of charge carriers. Synthesis of semiconductor nanocrystals (NC) has been intensely pursued due to their importance in optoelectronic, photovoltaic and biological applications. The applicability of the luminescent nanocrystals to the above application can be determined by their luminance quantum efficiency. Much effort has been endowed to enhance the quantum efficiency of semiconductor nanocrystals. The quantum efficiency of semiconductor nanocrystals is highly related with passivation of dangling bonds on the nanocrystal surface. Surface capping of nanocrystals with a wide band gap semiconductor can successfully improve the luminescence of nanocrystals and these core-shell NCs have shown to be generally more robust against chemical degradation or photo-oxidation (Xie et al., 2005). Such core-shell nanostructures reduce dangling bonds on nanocrystal surface by epitaxial growth of shell layers and are of practical interest for bio-imaging, LED, etc. Recently, semiconductor nanocrystals with quantum yield larger than $60 \%$ can be obtained with this core-shell approach. Basically, it needs that the core and shell materials should have similar crystal 
structure and lattice parameters to produce particles with high crystallinity without formation of interfacial defects. In order to improve quantum efficiency by improving crystallinity of nanocrystal through reducing interfacial lattice mismatch, multishell structure such as $\mathrm{CdS} / \mathrm{Zn}_{0.5} \mathrm{Cd}_{0.5} \mathrm{~S} / \mathrm{ZnS}$ was developed. Xie et al. synthesized multishell nanocrystals with high fluorescence quantum yield of 70-85\% (Xie et al., 2005). However, this core-shell approach requires a careful shell growth process using highly toxic and stench precursor such as diethyl zinc, trimethylsilyl sulfide, etc.

Recently, alloy nanocrystals with composition gradient along the radial direction were synthesized by several research groups using the difference in reactivity of cationic or anionic component (Bae et al., 2008; Bailey \& Nie, 2003). In particular, Bae et al. synthesized CdZnSeS nanocrystals by changing ratios of $\mathrm{Cd}$ to $\mathrm{Zn}$ and $\mathrm{Se}$ to $\mathrm{S}$ with the total concentrations of the Cd-Zn pair and Se-S pair fixed to be equal (Bae et al., 2008). The nanocrystals prepared in the single step synthesis are also structurally stable due to the chemical composition (or energy level) gradient in the radial direction, which effectively relieves the lattice mismatch and confines electrons and holes within the core.

As an alternative to II-VI semiconductors, various semiconductors with less-toxic elements have been proposed (Xu et al., 2008; Ryu et al., 2008; Zhong, 2008). Among these, CuInS 2 with bulk band gap of $1.5 \mathrm{eV}$ is selected as an important candidate for optical application. Recently, several works on the synthesis of $\mathrm{CuInS}_{2}$ (CIS) nanocrystals and efficient route to highly luminescent $\mathrm{CuInS}_{2} / \mathrm{ZnS}$ core shell nanocrystals were reported.5-7 Zhong et al. reported synthesis of $\mathrm{CuInS}_{2}$ nanocrystals using copper acetate, indium acetate and 1dodecanethiol by thermolysis of intermediate, CuIn(SR)x compound (Zhong, 2008). Moreover, Xie et al. reported synthesis of spherical CuInS 2 nanocrystals by modifying chemical reactivity of indium precursors and enhancement of their photoluminescence by formation of ZnS shell layers (Xie, 2008). However, inorganic shell capping of nanocrystals for high luminescence and photostability often requires repetitive formation of monolayers and precise process control. Recently, Ryu et al. reported a novel shell formation route for InP nanocrystals by refluxing with zinc acetate and 1-dodecanethiol (Ryu et al., 2009). The addition of zinc acetate played an important role in the formation of ZnS shell layers as well as in etching the defective surface of InP NCs by formation of acetic acid resulted from its reaction with long chain carboxylic acid. Moreover, Uehara et al. improve the photoluminescence of $\mathrm{CuInS}_{2}$ NCs by introducing lattice defects through highly offstoichiometric composition from $\mathrm{CuInS}_{2}$ (Uehara et al., 2008. This improvement in PL was attributed to the large population of donor or acceptor defects required for "donor-acceptor pair recombination (DAP)" of excited charge carriers. Recently, Hamanaka et al. assessed that the fluorescence in $\mathrm{CuInS}_{2} \mathrm{NCs}$ was originated from the deep surface trap recombination rather than DAP by considering the large Stokes shift of $\mathrm{CuInS}_{2} \mathrm{NCs}$ (Hamanaka et al., 2008). Thus, the origin of photoluminescence in CuInS 2 NCs is not fully investigated. We studied the photoluminescence of CuInS2-based nanocrystals with the time- and the temperature-dependent photoluminescence which provides the radiative and the non-radiative process in semiconductor nanocrystals. Herein, we developed a novel surface capping route for $\mathrm{CuInS}_{2} \mathrm{NCs}$, which is quite effective to remove surface trap sites and enhances the luminescence quantum yield. Furthermore, we reported the change of the dominant photoluminescence mechanism in $\mathrm{CuInS}_{2} \mathrm{NCs}$ by both controlling copper stoichiometry and surface modification. In addition to this, we report the tuning of emission wavelength by alloying. 


\subsection{Synthesis of highly luminescent $\mathrm{Cd}(\mathrm{Se}, \mathrm{S})$ alloy nanocrystals 3.1.1 Synthesis of nanocrystals}

The Cd precursor solution was synthesized by dissolving $8 \mathrm{mmol} \mathrm{CdO}$ to the mixture of $10 \mathrm{mmol}$ oleic acid and $20 \mathrm{ml} 1$-octadecene (ODE). Subsequently, the mixtures were heated to $150^{\circ} \mathrm{C}$ under $\mathrm{N}_{2}$ gas flow and metallic precursors were completely dissolved to form clear solution of metal oleate (solution A). Separately, selenium powders and sulfur powders with different ratio were dissolved in $2 \mathrm{ml}$ trioctylphosphine (TOP) and 10ml ODE under dry $\mathrm{N}_{2}$ atmosphere in the glove box maintaining the total amount of [Se]+[S] fixed and the mixed solution was swiftly injected to Cd-based precursor solution A at $300^{\circ} \mathrm{C}$. Aliquots were taken at different time interval after injection and rapidly cooled to room temperature. Before further characterization, acetone was added to precipitate CdSeS nanocrystals and the precipitates were separated and purified by repetition of centrifugation and redispersion to toluene.

\subsubsection{Nanocrystals with composition-gradient}

The synthesis of $\mathrm{CdSe}_{x} \mathrm{~S}_{\mathrm{y}}$ nanoparticles was carried out by a single injection of a premixed solution of Se/S in TOP and ODE solvents to the cadmium oleate solution heated at $300^{\circ} \mathrm{C}$. In this study, excess total cadmium relative to chalcogen $(\mathrm{Se}, \mathrm{S})$ ratio of $1 \sim 8$ times. Therefore Se and S precursors compete due to their difference in reactivity with $\mathrm{Cd}$. Then, following reactions are expected to take place:

$$
\begin{gathered}
\mathrm{Cd}(\mathrm{L}) n+\text { TOPSe } \rightarrow(\mathrm{CdSe})(\mathrm{L}) m(\mathrm{P}) \\
\mathrm{Cd}(\mathrm{L}) n+\mathrm{TOPS} \rightarrow(\mathrm{CdS})(\mathrm{L}) m(\mathrm{P}) \\
\mathrm{X}(\mathrm{CdSe})(\mathrm{L}) m(\mathrm{P}) p+\mathrm{Y}(\mathrm{CdS})(\mathrm{L}) m(\mathrm{P}) p \rightarrow \mathrm{CdSe} x \operatorname{s} y) m(\mathrm{P})
\end{gathered}
$$

where $\mathrm{L}=$ oleic acid and/or solvent, $\mathrm{P}=$ TOP, TOPS, or TOPSe, and $\mathrm{k}_{1}$ and $\mathrm{k}_{2}=$ rate constants of reactions 1 and 2. Reaction 1 and 2 is for formation of CdSe and CdS, respectively. The reaction 3 is for formation of alloy nanocrystals of CdSeS. It is known that $\mathrm{Cd}$ precursor reacted with Se faster than $\mathrm{S}$ to form CdSe preferably and leave surplus $\mathrm{S}$ after the reaction (Bailey et al., 2003).

Under Cd-rich conditions, the chalcogen precursors in with the limited supply would be completely consumed while the reactant in large excess would maintain a nearly constant concentration during the reaction and alloyed nanocrystals with a composition gradient structure can be produced (Bailey et al., 2003).

Fig. 3.1 shows photoluminescence (PL) spectra of CdSeS nanocrystals prepared with different cation to chalcogen concentration in the precursor solution. The peak intensity of PL spectrum grows to be intensified and the center of peak moves to low energy side. If the concentration of $\mathrm{Cd}$ and chalcogen precursors are similar or $[\mathrm{Cd}] /(\mathrm{Se}]+[\mathrm{S}]) \sim 1$, the concentration of $[\mathrm{Cd}]$ is changed with reaction time. In this case, the composition of nanocrystal is determined only by initial composition ratio of [Se] to [S] even the concentration of $\mathrm{Cd}$ in precursor solution changed with time and nearly homogeneous alloy of CdSeS can be obtained. Contrary to this, in the case of the large ratio of $\mathrm{Cd}$ to chalcogen or a large excess amount of cadmium precursors, the concentration of cadmium would be maintained without significant change during the reaction. Since Se is more reactive to $\mathrm{Cd}$ compared with S, Cd-Se bondings are formed in the initial stage of reaction. As the reaction 


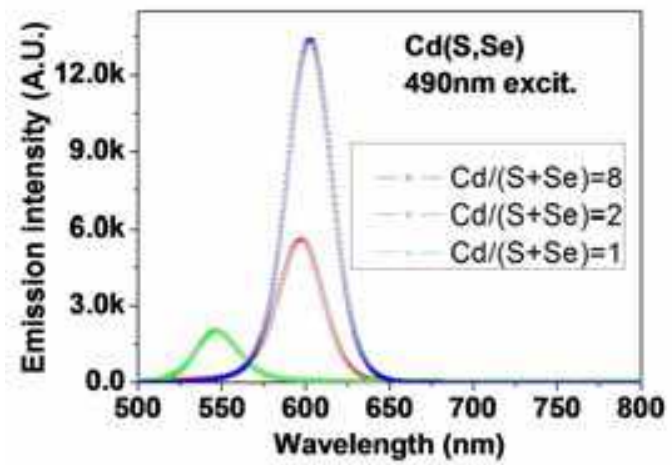

Fig. 3.1. Photoluminescence of CdSeS nanocrystal prepared with different ratio of [Cd] to $[\mathrm{Se}]+[\mathrm{S}]$

proceeds, the concentration of Se is depleted and number of Cd-S bondings is increases. After completion of reaction, cores of grown nanocrystals are Se-rich and the concentration of $S$ is increases along radial direction of nanocrystals. That is alloy nanocrystals with composition gradient are formed with this reaction conditions. Since the band gap of CdSe $\left(\mathrm{E}_{\mathrm{g}} \sim 1.8 \mathrm{eV}\right)$ is smaller than that of $\mathrm{CdS}\left(\mathrm{E}_{\mathrm{g}} \sim 2.3 \mathrm{eV}\right)$, the band gap of alloy nanocrystal grow larger along radial direction of nanocrystals. Then, a core-shell like nanostructures can be formed and luminescence of nanocrystal is expected to be enhanced under this condition. As shown in Fig. 3.1, the center of PL spectrum shifts to longer wavelength as the ratio of cation to chalcogen increases. In case of $[\mathrm{Cd}] /(\mathrm{Se}]+[\mathrm{S}]) \sim 1$, homogeneous alloy nanocrystals are expected to be formed and the peak position of the PL spectrum is positioned in short wavelength due to larger band gap of CdSeS alloy compared with CdSe. In case of $[\mathrm{Cd}] /(\mathrm{Se}]+[\mathrm{S}])>>1$, alloy nanocrystals with composition gradient along radial direction are expected to be formed and the peak position in the PL spectrum is determined by Se-rich composition of core of CdSeS alloy dots. In addition to this, since the composition gradient nanostructure in alloy nanocrystals provides effective confinement of electrons and holes, the intensity of the PL spectrum is highly enhanced. The maximum quantum yield of these alloy nanocrystals was larger than $90 \%$ by comparing photoluminescence of an organic dye, Rhodamine 6G.
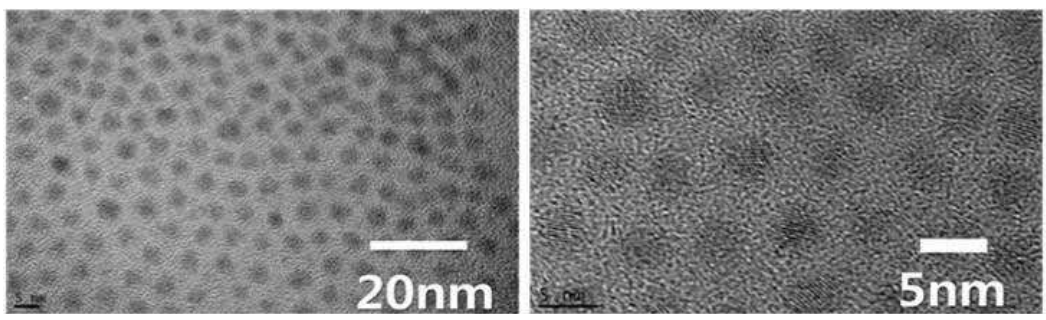

Fig. 3.2. TEM image of CdSeS nanocrystal grown for 2 minutes at $300^{\circ} \mathrm{C}$ under $\mathrm{N}_{2}$ flow with $[\mathrm{Cd}] /(\mathrm{Se}]+[\mathrm{S}])>>1$.

Fig. 3.2 shows TEM images of alloy nanocrystals grown with $[\mathrm{Cd}] /(\mathrm{Se}]+[\mathrm{S}])>>1$. Highly uniform and spherical nanocrystals with average size $4 \mathrm{~nm}$ were formed. The distance 
between surfaces of neighboring nanocrystals is about $2 \mathrm{~nm}$ which is similar to the length of oleic acid. Figure 3.3(a) shows powder diffraction profiles of CdSeS nancrystal grown with $[\mathrm{Cd}] /(\mathrm{Se}]+[\mathrm{S}])>>1$. The peak position of alloyed nanocrystals moved to higher angle which means smaller lattice parameters compared with CdSe. The calculated lattice parameters of grown nanocrystals are between that of CdSe and CdS, which confirms the formation of alloy nanocrystals and as the ratio of [Se] to [S] become smaller, the lattice parameter also become smaller. The maximum of photoluminescence of alloy nanocrystals can be changed by changing initial ratio of [Se] to [S] as shown in Fig. 3.3(b). That is, the luminescence wavelength can be tuned by changing initial ratio of [Se] to [S].
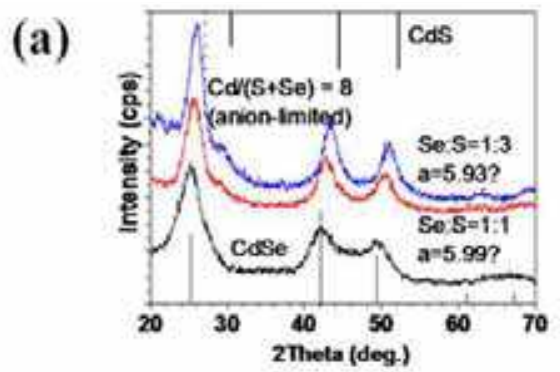

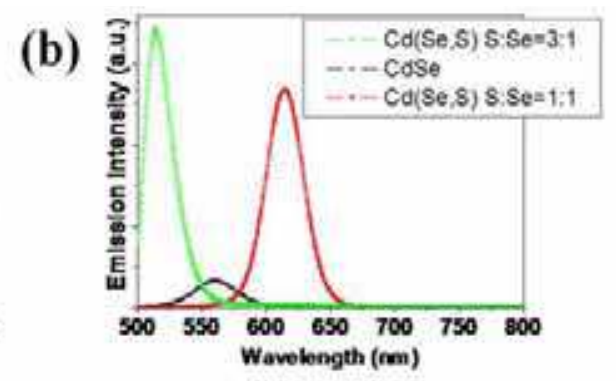

Fig. 3.3. (a) powder X-ray diffraction profiles (b) Photoluminescence spectra of CdSeS alloy nanocrystal synthesized with different ratio of [Se] to [S]
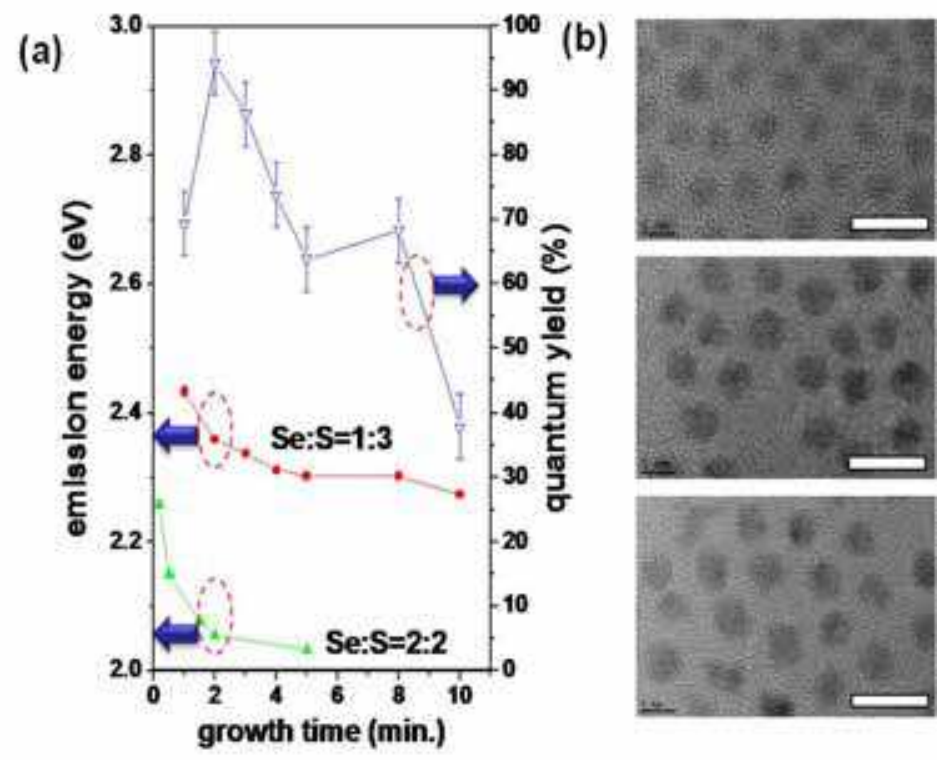

Fig. 3.4. (a) Temporal change of optical emission energy and quantum yield (b)TEM image of CdSeS alloy nanocrystals after 2 (upper), 4(middle), 8 minutes (lower). 
Figure 3.4 shows temporal development of photoluminescence spectra of $\mathrm{Cd}(\mathrm{Se}, \mathrm{S})$ alloy nanocrystals with initial Se-to-S ratio of 1:3. During the initial 2 minutes after injection of selenium precursor solution, the quantum yield of nanocrystals rapidly increased with time and photoluminescence spectra moves to lower energy side, which implies that "composition-gradient" structure is developed in the nanocrystals, which is structure composed of Se-rich core part and S-rich shell part. After 2 minutes at $300^{\circ} \mathrm{C}$, the quantum yield decreased and emission energy showed red-shift, monotonously. The quantum yield of nanocrystals rapidly decreased to be $65 \%$ and emission energy showed red-shift to be $2.3 \mathrm{eV}$ after 5 minutes. During this stage of reaction, nanocrystal shows slight size growth as shown in the TEM image of Fig. 4(b). The change in optical spectra and size of nanocrystals during the growth stage with time from 2 minutes to 5 minutes indicates destruction of "composition-gradient" structure and homogenization of Se and S distribution in the nanocrystals. At $300^{\circ} \mathrm{C}$, Se and S can diffuse into the other lattice site and exchange position. As a result of homogenization, high quantum yield obtained by CdS-rich surface layer was decreased. Further reaction at $300^{\circ} \mathrm{C}$ showed more rapid decrease in the quantum yield with small change in emission energy. TEM image showed some non-spherical and irregular nanocrystals. As shown in Fig. 3.5, photoluminescence spectra were rapidly broadened after 8 minutes at $300^{\circ} \mathrm{C}$. Li et al. showed that Ostwald ripening of the core and core/shell nanocrystals is inevitable at above $240^{\circ} \mathrm{C}$ ( $\mathrm{Li}$ et al, 2003). That is, broadening and photoluminescence spectra and irregular size distribution of nanocrystals indicates Ostwald ripening is dominant growth mechanism in this growth stage. The photoluminescence spectra of $\mathrm{Cd}(\mathrm{Se}, \mathrm{S})$ nanocrystals grown at $300^{\circ} \mathrm{C}$ show decrease after reaction for 2 minutes without significant change in their width. As mentioned earlier, one possible origin of this change in PL intensity is redistribution of Se and S atoms inside nanocrystals. It is inferred that a composition-gradient of Se and $S$ was formed in nanocrystals during the reaction at $300{ }^{\circ} \mathrm{C}$ for 2 minutes and the radial distribution of Se and S atoms in nanocrystals was homogenized by prolonged reaction at the same temperature by diffusion. For CdSe-based nanocrystals, the temperature of $300^{\circ} \mathrm{C}$ is sufficient to further diffusion of atoms in the nanocrystal. A schematic illustration shown in Fig. 3.6 described the redistribution of anions and Ostwald ripening during the prolonged reaction at $300^{\circ} \mathrm{C}$ inferred from the PL spectra.

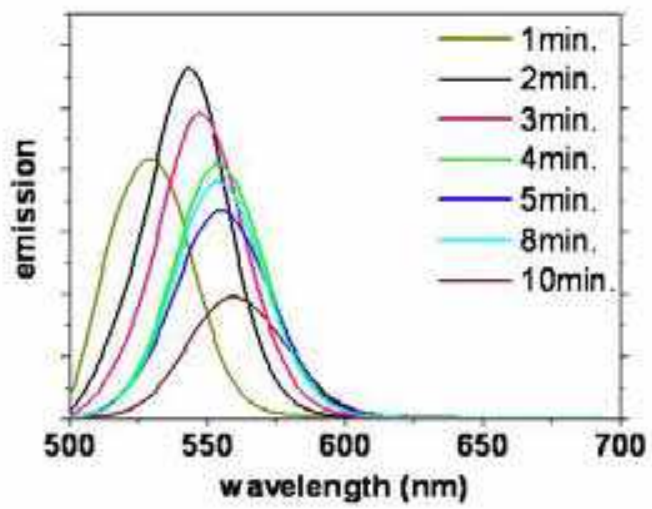

Fig. 3.5. Temporal change of photoluminescence spectra for $\mathrm{Cd}(\mathrm{Se}, \mathrm{S})$ nanocrystals grown at $300 \circ \mathrm{C}$. 

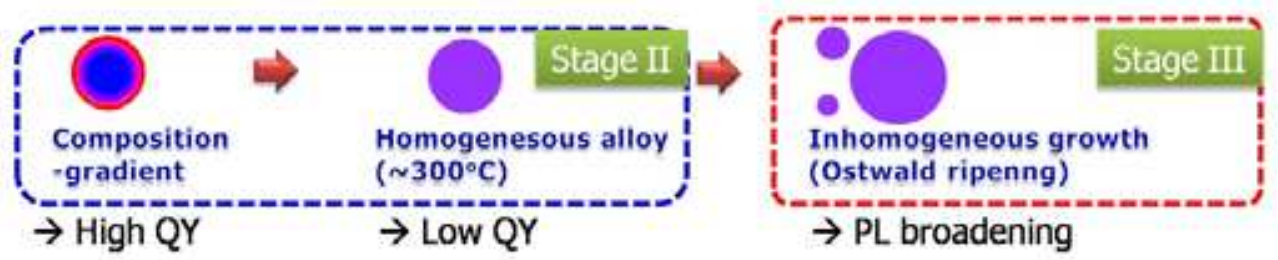

Fig. 3.6. A schematic illustration describing the origin of change in the PL spectrum for nanocrystals synthesized at $300^{\circ} \mathrm{C}$

This suggest that fast cooling after injection can retard homogenization of atomic distribution and conserve the composition-gradient in nanocrystals. Figure 3.7(a) shows temporal change of quantum yield of nanocrystals which were fast cooled to various temperature after injection of Se and S mixed precursor solutions at $300^{\circ} \mathrm{C}$. Without fast cooling to lower temperature, the quantum yield of nanocrystals initially reached to maximum value and rapidly diminished to be less than $40 \%$ after 8 minutes. In contrast to this, nanocrystals fast cooled to $225^{\circ} \mathrm{C}$ after precursor injection showed no significant decrease during 30 minutes. Moreover, the PL spectra for nanocrystals reacted at higher temperature showed clear red shift and broadening contrary to those reacted at lower temperature as displayed in Fig 3.7(b).
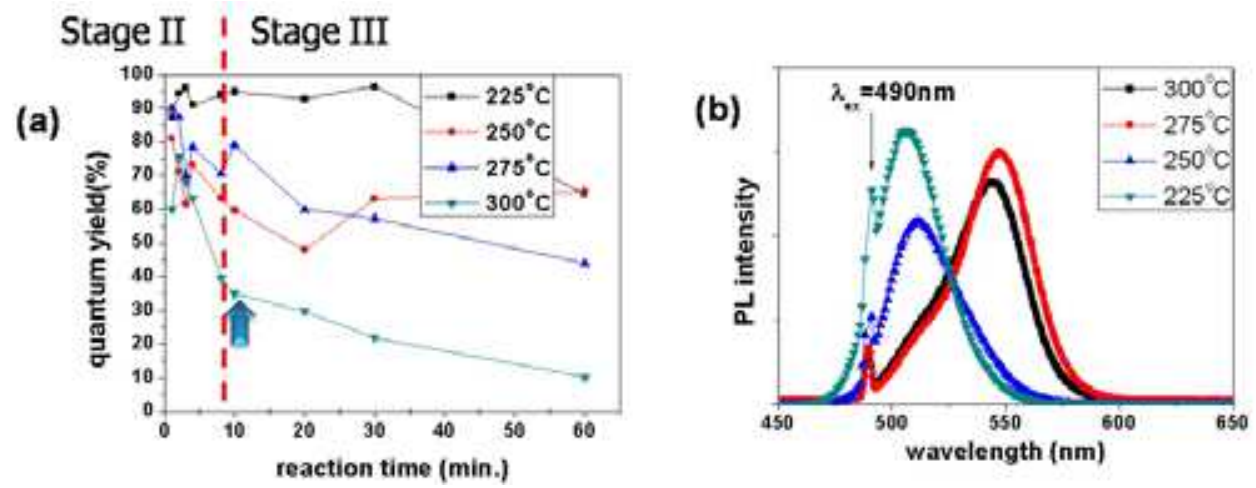

Fig. 3.7. Temporal change of (a) quantum yield and (b) photoluminescence spectra for $\mathrm{Cd}(\mathrm{Se}, \mathrm{S})$ nanocrystals grown at various temperatures for 20 minutes.

\subsection{Photoluminescence of Culn $S_{2}$ nanocrystals: Effect of alloying 3.2.1 Synthesis of CulnS $S_{2}$ nanocrystals}

The CuInS 2 NCs were synthesized using copper acetate, indium acetate and 1-dodecanethiol (DDT) according to the protocol reported by Zhong et al (Zhong et al., 2008 ). In detail, the amount of $\mathrm{Cu}(\mathrm{II})$ acetate was varied from $0.2 \mathrm{mmol}$ to $1 \mathrm{mmol}$ and indium acetate with quantity of $1 \mathrm{mmol}$ was used. Those precursors were dispersed in $30 \mathrm{ml}$ of 1 -octadecene under inert atmosphere at room temperature and subsequently heated to $240^{\circ} \mathrm{C}$. After reaction for 15 minutes, the solution was cooled to room temperature. To construct coreshell structure, $1 \mathrm{mmol}$ of zinc acetate was added to the $\mathrm{CuInS}_{2}$-based core solution after cooling to room temperature without purification and separation of synthesized NCs. In 
some experiment, we substitute cadmium acetate for small part of zinc acetate maintaining total amount of metal acetate constant to be $1 \mathrm{mmol}$. For colloidal stability of NCs, $3 \mathrm{mmol}$ of palmitic acid mixed with $10 \mathrm{ml}$ of 1 -octadecene was also added to the solution. Subsequently, the mixture was heated to $230^{\circ} \mathrm{C}$ under inert $\mathrm{N}_{2}$ atmosphere and reacted without further addition of precursors. The mixed solution became uniform at above $120^{\circ} \mathrm{C}$ and further dwelling at $230^{\circ} \mathrm{C}$ to enhance the luminescence. After refluxing for 5 hours, DDT with quantity equivalent to zinc acetate was injected to the solution and maintained for an hour to form $\mathrm{ZnS}$ shell at the same temperature. Aliquots were taken at the end of each steps and rapidly cooled to room temperature. Before further characterization, polar solvent such as acetone, methyl alcohol, etc. was added to precipitate $\mathrm{Cu}-\mathrm{In}-\mathrm{S}_{2}$-based NCs and the precipitates were purified by repetition of centrifugation and re-dispersion to n-hexane.

\subsubsection{Effect of composition and surface modification}

Reports have shown that the main fluorescence process in CIS NCs is closely related to lattice defects such as copper vacancy, indium substitute anti-site defect, etc.(Uehara et al., 2008). This implies that controlling crystal defect can enhance the fluorescence of $\mathrm{CuInS}_{2}$ nanocrystals and intentional formation of some crystal defects was proved to be efficient to high fluorescence quantum yield. Fig. 3.8(a) shows various fluorescence spectra obtained from $\mathrm{Cu}_{1-\mathrm{x}} \mathrm{InS}_{2}(\mathrm{x}=0 \sim 0.8)$ NCs with various different starting compositions.
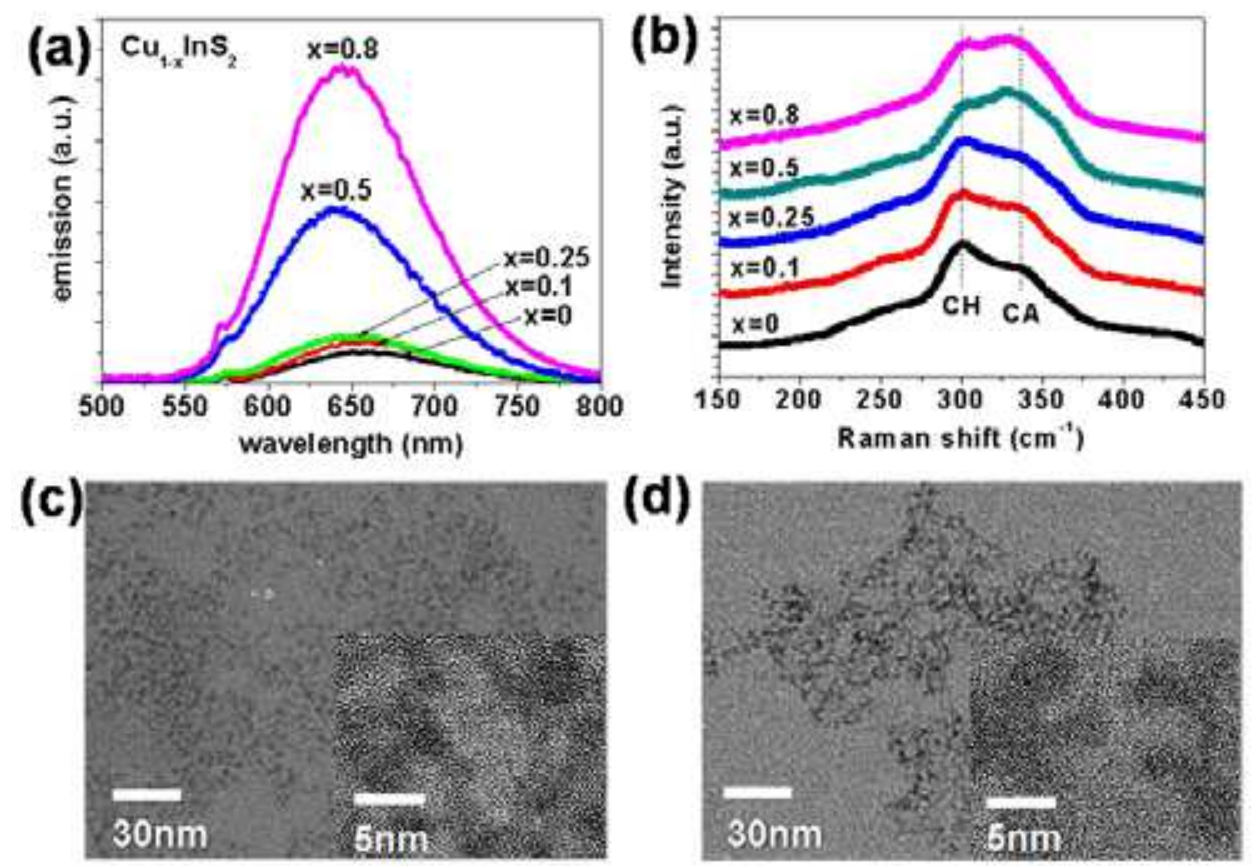

Fig. 3.8. Photoluminescence (PL) and (b) Raman spectra of $\mathrm{Cu}_{1-x} \mathrm{InS}_{2}$ nanocrystals prepared with different degree of copper deficiency in the starting solutions. (c-d) TEM images of $\mathrm{Cu}_{1-}$ ${ }_{x} I n S_{2}$ nanocrystals with different starting composition of $x=0$ and $x=0.8$, respectively. 
The luminescence intensity of CIS NC was highly enhanced and the wavelength of emission spectra showed blue-shift with minimizing copper content in the starting solution. As mentioned earlier, incorporation of crystal defect in CIS NCs by highly Cu-deficient composition in the starting solution can be ascribed to the source of improved luminescence. Uehara et al. explained the blue-shift of fluorescent spectra by the lowering of valence band due to Cu-deficiency in CIS NCs. The composition of CIS NCs prepared from precursor solution with $\mathrm{Cu}: \mathrm{In}=0.2: 1$ was measured to be $\mathrm{Cu}: \mathrm{In}=0.19: 1$ from induction-coupled plasmamass spectrometry (ICP-MS). Thus, Cu-deficiency in starting solution resulted in large $\mathrm{Cu}$ deficiency in CIS NCs. In addition, the Raman spectra of Cu-deficient CIS NCs displayed in Fig. 3.8(b) show a characteristic peak of "CuAu"-type ordering (CA) at $340 \mathrm{~cm}^{-1}$ as well as

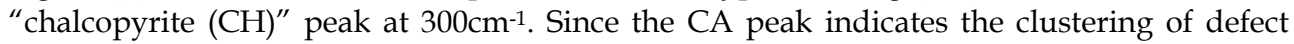
pair at high concentration of defects (Uehara et al., 2008;Alvarez-Garcia et al., 2005), CA peak in the Raman spectrum of Cu-deficient Cu-In-S NCs also supports a large number of crystal defects induced by copper deficiency in the starting solutions. In particular, the more

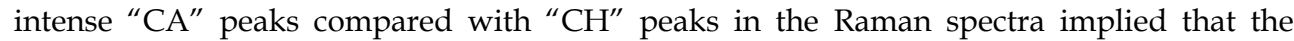
highly Cu-deficient composition in the starting solution gave rise to higher concentration of lattice defects in the synthesized NCs. As observed in the TEM image in Fig. 3.8(c) \& (d), the grown NCs was quite irregular in shapes and showed broad size distribution irrespective of $\mathrm{Cu}$-deficiency in the starting solutions.

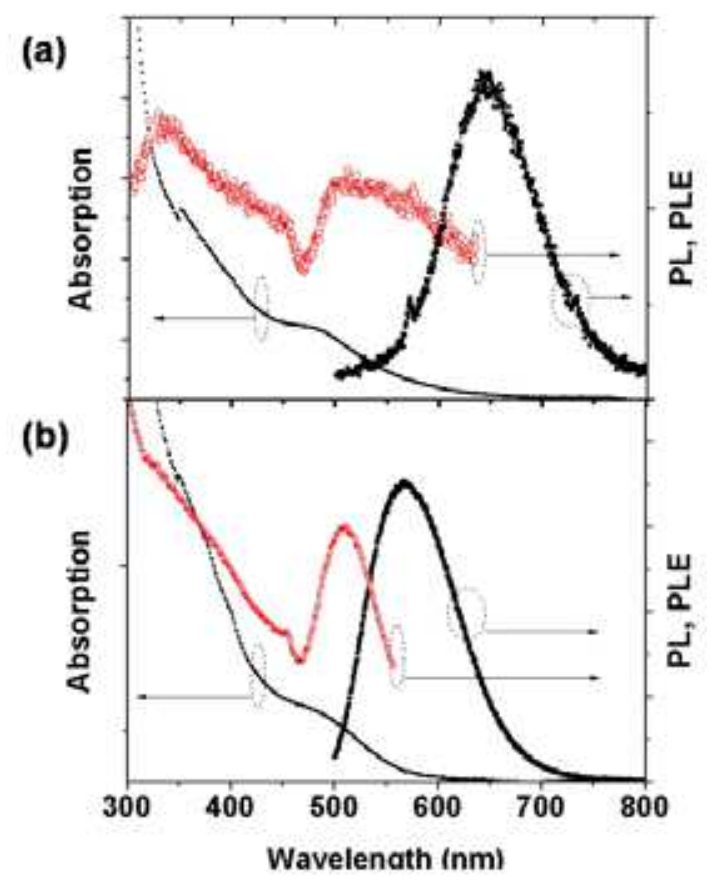

Fig. 3.9. Absorption, PL and PLE of (a) as-synthesized $\mathrm{Cu}_{1-\mathrm{x}} \mathrm{InS}_{2}$ nanocrystals $(x=0.2)$ and (b) $\mathrm{Cu}_{1-\mathrm{x}} \mathrm{InS}_{2}$ nanocrystals $(\mathrm{x}=0.2)$ after refluxing with zinc acetate and palmitic acid for 5 hours at $230^{\circ} \mathrm{C}$. 
According to Zhong et al.'s work, CuInS 2 NCs were synthesized from the thermal decomposition of intermediate compound, $\mathrm{CuIn}\left(\mathrm{SC}_{12} \mathrm{H}_{25}\right)_{\times}$complex and prepared NCs were highly aggregated due to the oriented attachment of small particles (Zhong et al., 2008). As a result of irregular shape and poly-dispersed size distribution, the absorption spectrum of NCs showed broad shoulder with long wavelength trail and photoluminescence excitation spectrum (PLE) showed broad intensity distribution shown in Fig. 3.9(a).

The non-radiative recombination of electron-hole pairs by the surface states has been attributed to the major origin of the low quantum yield in semiconductor NCs. In addition, the passivation of surface defects by further shell growth on the semiconductor NCs was proved to be effective for high luminescence properties. Ryu et al. have suggested that zinc acetate acts as a source of ZnS shell on InP and acetic acid produced from dissociation of zinc acetate etches the defective surface of as-synthesized InP cores (Ryu et al., 2009). In this study, impressed by Ryu et al.'s work, we tried to improve the photoluminescence (PL) of CIS NCs by modifying their surface through refluxing with zinc acetate and palmitic acid. Fig. 3.9(b) shows optical spectra of CIS NCs after treatment with zinc acetate and palmitic acid for 5 hours at $230^{\circ} \mathrm{C}$. The long wavelength trail in the absorption spectrum was efficiently suppressed and photoluminescence excitation spectrum (PLE) showed narrow intensity distribution. Since the long wavelength trail in the absorption spectra and broad PLE is mainly caused by inhomogeneous size distribution, more uniform size distribution of NCs was obtained after refluxing with zinc acetate and palmitic acid as inferred from the narrow absorption band in the optical spectra.

In addition, TEM images of NCs shown in Fig. 3.10(a) also support narrowing of the size distribution after refluxing with zinc acetate and palmitic acid. The low resolution image showed equally spaced dots, and oblate nanocrystals with crystalline lattice was observed from the high resolution image. Thus, the refluxing of CIS core solution with zinc acetate and palmitic acid resulted in a digestive ripening of CIS NCs. The aggregated CIS NCs were supposed to be broken into quasi-monodispersed NCs by etching with acetic acid produced from the reaction between zinc acetate and palmitic acid. Fig. 3.10(b) shows temporal evolution of the photoluminescence spectra of CIS NCs after refluxing with zinc acetate and palmitic acid at $230^{\circ} \mathrm{C}$. The fluorescence quantum yield of NCs was highly enhanced to be above $40 \%$, which was initially $3 \%$ for pristine CIS NCs. In particular, the quantum yield was improved up to $70 \%$ by further injection of DDT and subsequent refluxing for an hour at the same temperature. Since CIS core solution contained large amount of sulfur to suppress $\mathrm{Cu}_{\mathrm{x}} \mathrm{S}$ formation (Zhong et al., 2008) and as-synthesized CIS cores were refluxed without further purification or removing unreacted precursors, formation of ZnS shell is expected even without further injection of sulfur precursor such as DDT during the refluxing. In addition, the crystal structure of $\mathrm{CuInS}_{2}$ and $\mathrm{ZnS}$ is quite similar and lattice mismatch between them is about $2 \%$. Thus, as a result of passivation of surface defects and confinement of charge carriers by coherent shell formation, fluorescence of the CIS NCs was intensified by simple refluxing with zinc acetate and palmitic acid. Moreover, we supposed that etching of defect-rich surface with acetic acid also contributed to high quantum yield in CIS NCs refluxed with zinc acetate and palmitic acid. We have checked the effect of Cudeficiency on the luminescence of CIS/ZnS NCs. Fig. 3.10(c) shows PL spectra of CIS/ZnS NCs prepared with different copper content in the starting solutions. The quantum yield of $\mathrm{Cu}_{1-\mathrm{x}} \mathrm{InS}_{2} / \mathrm{ZnS} \mathrm{NCs}$ was measured to be $3 \%, 16 \%, 70 \%$ for $\mathrm{x}=0, \mathrm{x}=0.5, \mathrm{x}=0.8$, respectively. This implies that refluxing CIS NCs with zinc acetate and palmitic acid does not remove 

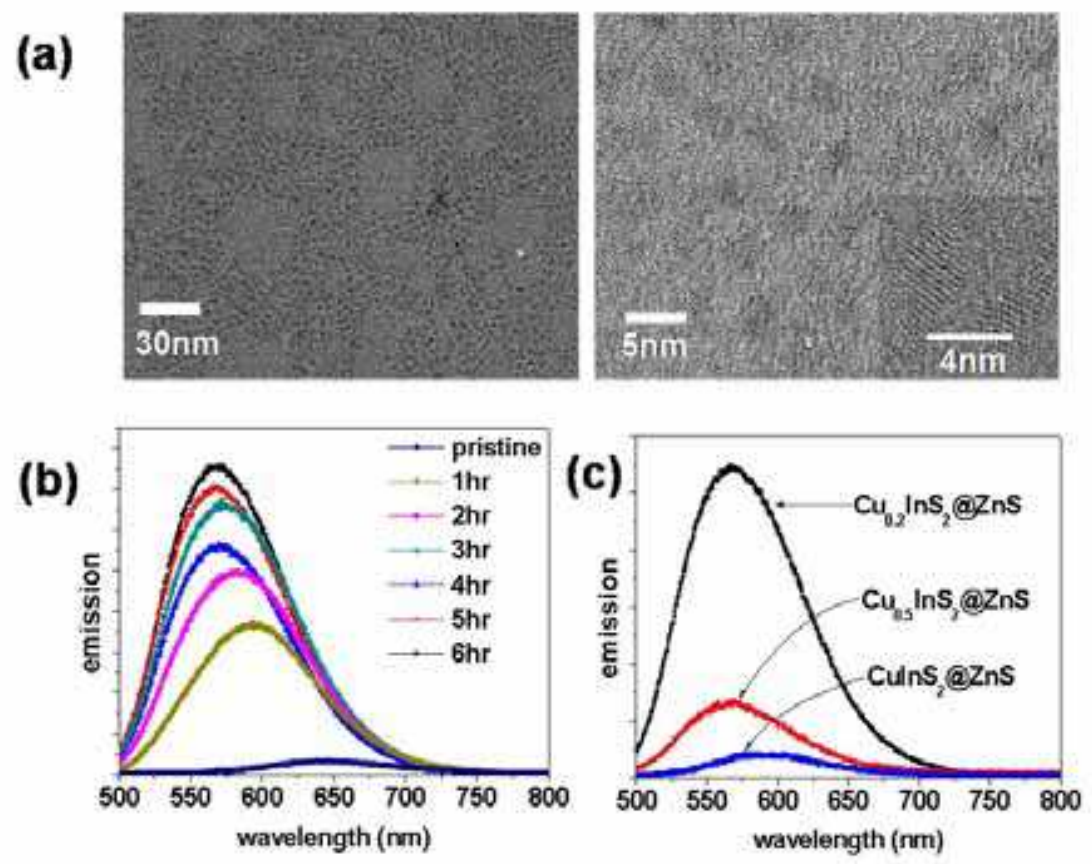

Fig. 3.10. (a) TEM images of $\mathrm{Cu}_{1-x} \mathrm{InS}_{2}$ nanocrystals $(x=0.8)$ treated with zinc acetate and palmitic acid for 5 hours at $230^{\circ} \mathrm{C}$. (b) Temporal evolution of PL of $\mathrm{Cu}_{1-\mathrm{x}} \mathrm{InS}_{2}$ nanocrystals $(x=0.2)(c) P L$ of $\mathrm{Cu}_{1-\mathrm{x}} \mathrm{InS}_{2} \mathrm{NCs}$ with various starting composition $(\mathrm{x}=0,0.5,0.8)$ after refluxing with zinc acetate and palmitic acid for 5 hours at $230^{\circ} \mathrm{C}$.

copper defects located in the interior of CIS NCs, but passivate surface defects of the nanocrystals. Thus, it is concluded that Cu-deficiency is essential to high quantum yield of CIS NCs even after inorganic capping with ZnS.

As mentioned earlier, the origin of photoluminescence in copper indium sulfide nanocrystals was not unambiguously determined. In this work, we investigated the photoluminescence of nanocrystals with time-decay PL to obtain the detailed information on the phtoluminescence process. PL dynamics contains information about the recombination of photoinduced carriers in the nanocrystals. Fig. 3.11(a) demonstrates timedecay PL data of CIS NCs with various copper deficiency. The nanocrystals with small copper deficiency showed a much rapid decay contrary to those with large copper deficiency. In contrast to this, the surface modification of CIS NCs decreased their PL decay rate. In order to analyze the time-decay PL more quantitatively, we analyzed the multiexponential PL decay by reconstructing the distribution of decay times with a minimum of $a$ priori assumptions on the PL decay (Schlegel et al., 2002; Zenkevich et al., 2005). Since the recovery of decay time distribution from the decay data is an ill-posed problem, a regularization method should be used. We analyzed the multi-exponential PL decay by reconstructing the distribution of decay times with Tikhonov regularization method (Kapitonov et al., 1999; Hansen, 1994). Decay time distribution was computed using n=400 exponential terms with decay times uniformly spaced on the logarithmic scale. Fig. 3.11(b) 

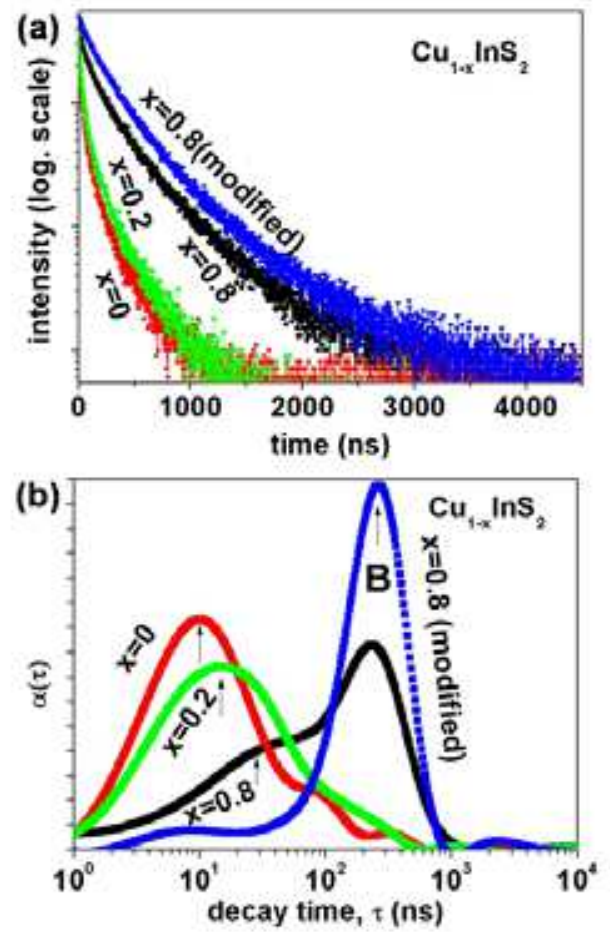

Fig. 3.11. (a) Time-dependent photoluminescence and (b) reconstructed decay time distribution of $\mathrm{Cu}_{1-\mathrm{x}} \mathrm{InS}_{2}$ nanocrystals with various copper deficiency $(\mathrm{x})$ and the surface modified $\mathrm{Cu}_{1-\mathrm{x}} \mathrm{InS}_{2}(\mathrm{x}=0.8)$ nanocrystals.

showed decay time distributions reconstructed from the time-resolved PL data. It is known that the decay time distribution in the photoluminescence of semiconductor nanocrystals can be modeled by log-normal statistics (van Driel et al., 2007). The decay time distribution for CIS NCs with small copper deficiency exhibited a log-normal distribution centered at $\tau_{\max }=3 \mathrm{~ns}$ and for $\mathrm{x}=0$ and $\tau_{\max }=15 \mathrm{~ns}$ for $\mathrm{x}=0.2$. In contrast to this, a bimodal decay time distribution was shown at $\tau_{\max }=35 \mathrm{~ns}$ and $\tau_{\max }=237 \mathrm{~ns}$ for $\mathrm{x}=0.8$. That is, the contribution of the fast decay component was highly reduced and the slow decay component was dominant for the copper-deficient CIS NCs. Thus, the reconstruction of decay time distribution with a regularization method provides more detailed information on the excited state decay process compared with direct numerical fitting with stretched exponential function. In addition, the fast decay component in the decay time distribution was highly minimized by the surface modification of copper-deficient CIS NCs through formation of ZnS shell and etching of their surface.

It is noted that the decay time component of initially populated core-state recombination of exciton was known to be about 3ns (Dean, 1973). In addition, the large Stokes shift and the broad PL spectra eliminate the possibility of the direct exciton recombination in CIS NCs. The suppression of the fast decay component in the time-resolved PL by the surface 
modification of CIS NCs strongly indicated a contribution of trap-related emission. The decay time of PL in the semiconductor nanocrystals in the range of 15 through $25 \mathrm{~ns}$ at room temperature was often ascribed to trap-related emission as reported for CdSe and CdSe/ZnS (Wang et al., 2003; Schlegel et al., 2002). The broad distribution of decay time for the fast decay component possibly resulted from the dispersion in trap energy levels. Thus, it is concluded that both trap-related emission and donor-acceptor recombination contribute to the luminescence of the CIS NCs and emission from the latter process is dominant for the CIS NCs with large Cu deficiency.

The role of trap states was further evidenced by the temperature-dependent photoluminescence spectra shown in Fig. 3.12(a). The PL spectra of CIS NCs with $x=0.8$ was quenched when the temperature was increased at above $100 \mathrm{~K}$. Contrary to this, PL spectra of the surface modified CIS NCs remain constant at least up to $300 \mathrm{~K}$. It is known that the quenching of the emission occurs at high temperature by ionization of the trapping centers if trapped electrons or holes play a role in the non-radiative recombination. Then, the thermal activation energy of quenching process can be closely related to the ionization energy of the trapping centers (Binsma et al., 1982). The thermal change in photoluminescence of semiconductor were often described as eq(1) which considers capture cross-section of carriers at the recombination centers (Krustok et al., 2000):

$$
I(T)=I_{0} /\left(1+\phi_{1} T^{3 / 2}+\phi_{2} T^{3 / 2} \exp \left(-E_{T} / k_{B} T\right)\right)
$$

Here, $I_{0}, \phi_{1}, \phi_{2}$ are the three fitting parameters and $E_{T}$ is the thermal quenching energy. By numerical fitting with eq(1), the thermal change of the integrated PL intensity of bare CIS NCs was well fitted and the thermal quenching energy of bare CIS NCs was estimated to be $84 \mathrm{meV}$. Thus, the temperature-dependent PL study showed that the trap states incorporated in the uncapped CIS NCs were effectively removed by the surface modification through refluxing with zinc acetate and palmitic acid.
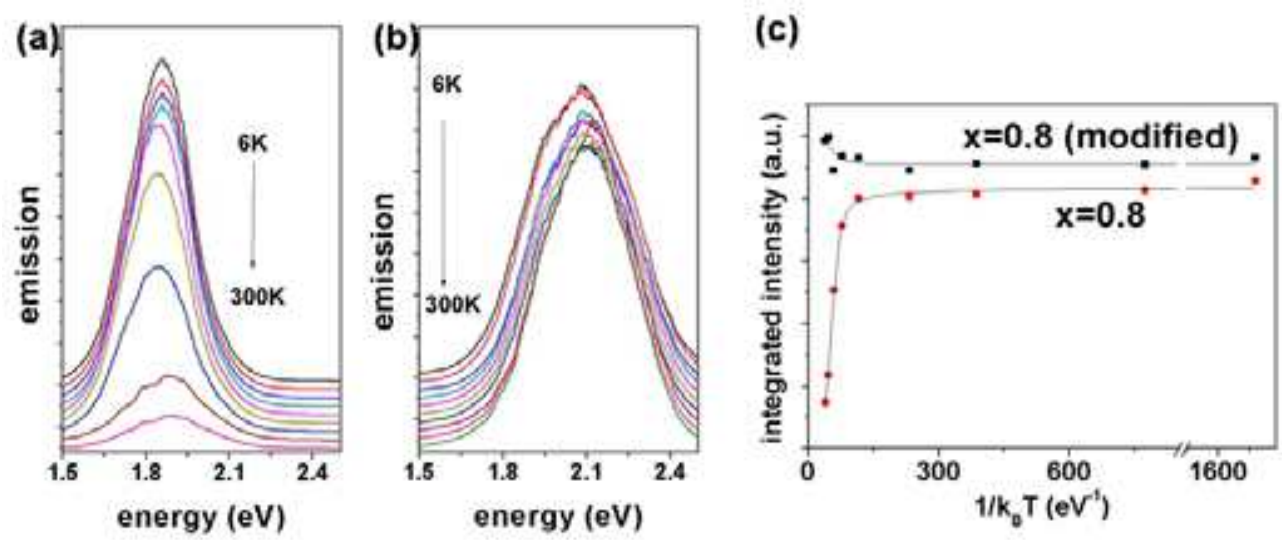

Fig. 3.12. Temperature-dependent photoluminescence of (a) $\mathrm{Cu}_{1-x} \operatorname{InS}_{2}$ nanocrystals $(x=0.8)$ and (b) the surface modified $\mathrm{Cu}_{1-\mathrm{x}} \mathrm{InS}_{2}(\mathrm{x}=0.8)$ nanocrystals; (c) Thermal change of the integrated intensity of photoluminescence spectra of both the as-synthesized and the surface modified $\mathrm{Cu}_{1-\mathrm{x}} \mathrm{InS}_{2}(\mathrm{x}=0.8)$ nanocrystals. 


\subsubsection{Effect of alloying on the photoluminescence of $\mathrm{CuInS}_{2} / \mathrm{ZnS}$ nanocrystals}

One of important features in copper deficient CIS/ZnS core-shell nanocrystals is large blueshift of luminescence intensity after refluxing nanocrystals with zinc acetate and palmitic acid. In contrast to digestive ripening of CdSe quantum dots demonstrating red-shift in PL spectra, digestive ripening of CIS NCs by refluxing with zinc acetate and palmitic acid showed blue-shift in PL spectra. Fig. 3.10(b) shows temporal development of photoluminescence excitation spectra of CIS NCs during the surface treatment with zinc acetate and palmitc acid. The blue shift of luminescence peak implies that the band gap of CIS NCs increases with increasing reflux time. Several reports have suggested various origin of blue-shift in PL such as size reduction by etching with acetic acid (Ryu et al., 2009), alloying of CIS NCs with ZnS (Uehara et al., 2008) and strain field caused by lattice mismatch between core material and shell material in the "core/shell" NCs. In order to test the feasibility of these suggestions, we refluxed $\mathrm{Cu}_{1-\mathrm{x}} \operatorname{InS}_{2}(\mathrm{x}=0.8)$ core solution with the cadmium acetate as well as zinc acetate and palmitic acid. Fig. 3.13(a) showed the PL spectra and their temporal evolution during refluxing. Since the total amount of metal acetates added was maintained irrespective of the relative portion of cadmium acetate in the reaction mixture, the amount of free acetic acid generated from reaction between metal acetate and palmitic acid can be considered to be constant. Hence, if the contribution of size reduction by etching with acetic acid is significant, addition of cadmium acetate is hard to modify the blueshift in PL. However, as the amount of cadmium acetate was increased, the blue shift of emission wavelength was minimized. In particular, the blueshift in PL after refluxing at $230^{\circ} \mathrm{C}$ was effectively suppressed by applying equal amount of cadmium acetate and zinc acetate. Thus, the influence of etching with acetic acid on the shift in the fluorescence wavelength is negligible. In addition, possibility of the contribution of alloying with wider band gap semiconductor can be eliminated. Since both CdS (Bulk Eg=2.4eV) and ZnS (Bulk $\mathrm{Eg}=3.6 \mathrm{eV}$ ) have much wide band gap compared with $\mathrm{CuInS}_{2}(\mathrm{Bulk} \mathrm{Eg}=1.5 \mathrm{eV})$, alloying or doping of $\mathrm{Cd}$ or $\mathrm{Zn}$ into the lattice of CIS NCs usually exhibit clear blue-shift in PL. Then, the contribution of alloying to the blue-shift in PL observed in this study is not plausible.

It is known that lattice strain can change band gap energy significantly by a coherent overgrowth of shell material on a small core (Smith \& Mohs, 2009). The lattice mismatch between core and shell of nanocrystals was estimated to be about $2 \%$ for CIS/ZnS NCs. The lattice stress applied to a CIS core by a ZnS shell overgrowth is compressive as estimated from lattice parameters of each materials (CIS: $a=0.5517 \mathrm{~nm}, \mathrm{ZnS}: \mathrm{a}=0.5345 \mathrm{~nm}$ ). The values of band deformation potential (in $\mathrm{eV} /$ unit dilatation) for CIS are known to be -11.0 and +8.27 for conduction band and valence band, respectively (Quintero et al., 1992). Then, energy gap of CIS changed to $-19.27 \mathrm{eV}$ by unit dilatation. The compressive stress applied to CIS NCs induces contraction of CIS lattice and increase the band gap. As a result of this, the PL spectra shifted to high energy or short wavelength. In addition, stress applied to a CIS core by a CdS shell is tensile considering lattice parameter of CdS $(a=5.820 \mathrm{~nm})$. Then, the position of PL spectra moves to low energy or long wavelength by capping with CdS. In fact, CdS capping on $\mathrm{CuInS}_{2}$ NCs showed clear redshift in PL spectra.Thus, the position of PL spectra for CIS NCs refluxed with mixture of cadmium acetate, zinc acetate and palmitc acid should move to long wavelength since sign of stress on CIS NCs by capping changes from compressive to tensile through increasing amount of cadmium acetate. Hence, it is concluded that the noticeable blue shift in PL spectra of CIS NCs after refluxing with zinc acetate and palmitic acid is a result of lattice strain applied to CIS cores by formation of inorganic shell layers. 

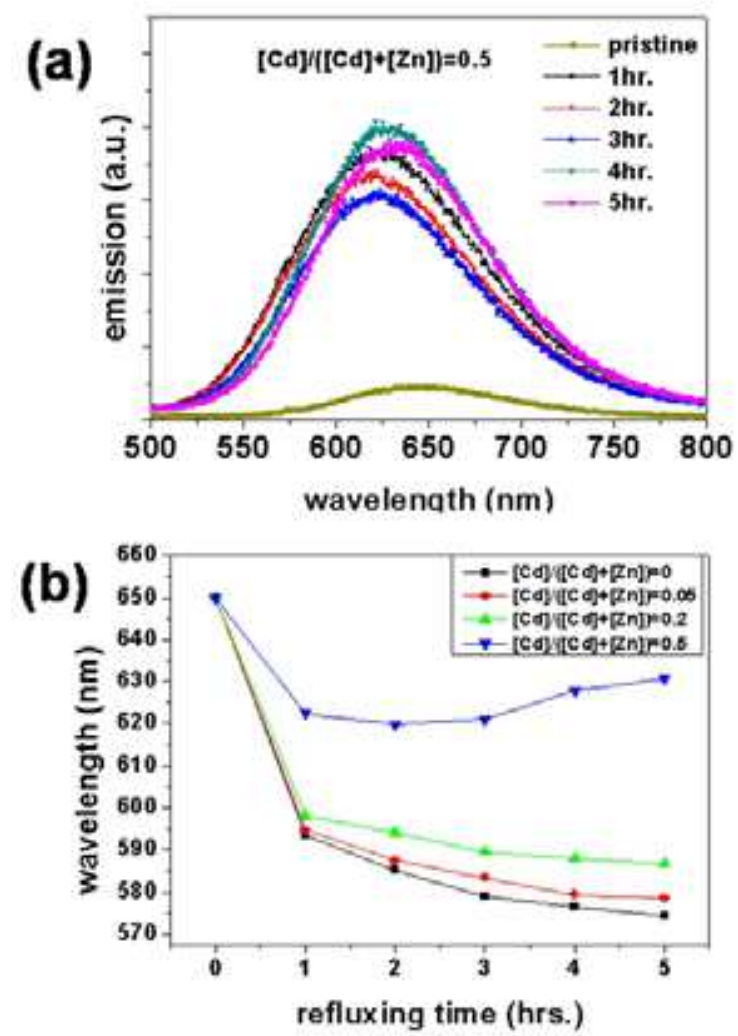

Fig. 3.13. Temporal change of (a) photoluminescence spectra and (b) emission wavelength of $\mathrm{Cu}_{1-\mathrm{x}} \mathrm{InS}_{2}$ nanocrystals $(\mathrm{x}=0.8)$ the surface modified with alloyed capping layers.

\section{References}

Alivisatos, A. P. (1996). Semiconductor Clusters, Nanocrystals, and Quantum Dots, Science Vol. 271, pp.933-937

Alvarez-Garcia, J.; Barcones, B.; Perez-Rodrigues, A.; Romano-Rodrigues, A.; Morante, J. R.; Janotti, A; Wei, S. -H. \& Scheer, R. (2005). Vibrational and crystalline properties of polymorphic CuInC 2 (C=Se,S) chalcogenides, Phys. Rev. B, Vol. 71, 054303.

Asokan, S.; Krueger, K. M.; Alkhawaldeh, A.; Carreon, A. R.; Mu, Z.; Colvin, V. L.; Mantzaris, N. V. \&Wong, M. S. (2005). The use of heat transfer fluids in the synthesis of high-quality CdSe quantum dots, core/shell quantum dots, and quantum rods, Nanotechnology Vol. 16, pp. 2000-2011

Asokan, S.; Krueger, K. M.; Colvin, V. L. \& Wong, M. S. (2007) Shape-Controlled Synthesis of CdSe Tetrapods Using Cationic Surfactant Ligands, Small Vol. 3, pp. 1164-1169 
Bae, W. K.; Char, K. H.; Hur H. \& Lee, S. (2008). Single-Step Synthesis of Quantum Dots with Chemical Composition Gradients Chem. Mater. Vol. 20, pp.531-539

Bailey, R. E. \& Nie, S. M. (2003). Alloyed Semiconductor Quantum Dots: Tuning the Optical Properties without Changing the Particle Size, I Am. Chem. Soc. Vol. 125, pp. 71007106

Bawendi, M.; Steigerwald, M. \& Brus, L. (1990). The Quantum Mechanics of Larger Semiconductor Clusters, Annu. Rev. Phys. Chem. Vol. 41, pp. 477-496

Binsma, J.; Giling, L.J.; Bloem, J. (1982). Luminescence of CuInS 2 : I. The broad band emission and its dependence on the defect chemistry, el Lumin. , Vol. 27 , pp. 35-53.

Comas, F.; Trallero-Giner, C.; Studart, N. \& Marques, G. E. (2002). Interface optical phonons in spheroidal dots: Raman selection rules Phys. Rev. B Vol. 65, pp. 073303-073305

Dalpian, Gustavo M. \& Chelikowsky, J.R. (2006). Self-Purification in Semiconductor Nanocrystals, Phys. Rev. Lett., Vol. 96, pp. 226802

Dean, P.J. (1973). in Progress in Solid State Chemistry McCaldin, J.O., Somorjai, G.(Eds), Pergamon: New York, Vol. 8 p. 1-126.

van Driel, A.F.; Nikolaev, S.; Vergeer, P.; Lodahl, P.; Vanmaekelbergh, D.\& Vos, W.L. (2007). Statistical analysis of time-resolved emission from ensembles of semiconductor quantum dots: Interpretation of exponential decay models, Phys. Rev. B., Vol. 75, 035329.

Erwin, S. C.; Zu, L. J.; Haftel, M. I.; Efros, A. L.; Kennedy, T. A. \& Norris, D. J. (2005) Doped Nanocrystals, Nature Vol. 436, pp. 91-94

Hamanaka,Y.; Kuzuya,T.; Sofue,T.; Kino, T.; Ito, K. \& Sugiyama, K. (2008). Defect-induced photoluminescence and third-order nonlinear optical response of chemically synthesized chalcopyrite CuInS2 nanoparticles, Chem. Phys. Lett. Vol. 466, pp.176180

Hankare, P. P.; Delekar, S. D.; Chate, P. A.; Sabane, S. D.; Garadkar, K. M. \& Bhuse, V. M. (2005). A novel route to synthesize $\mathrm{Cd}_{1-x} \mathrm{~Pb}_{x} \mathrm{Se}$ thin films from solution phase, Semicond. Sci. Technol. Vol. 20, pp. 257-264

Hansen P. C. (1994). Regularization Tools: A Matlab package for analysis and solution of discrete ill-posed problems, Numerical Algorithms, Vol.6, pp. 1-35

Hsu, Y. \& Lu, S. (2008) Dopant-Induced Formation of Branched CdS Nanocrystals, Small, Vol. 4, pp. 951-955

Hofhuis, J.; Schoonman, J.; Goossens, A. (2008). el Phys. Chem. C, Vol. 112, pp.15052-15059.

Jing, P.; Zheng, J.; Ikezawa, M.; Liu, X.; Lv, S.; Kong, X.; Zhao, J. \& Masumoto, Y. (2009). Temperature-Dependent Photoluminescence of CdSe-Core CdS/CdZnS/ZnSMultishell Quantum Dots, el Phys. Chem. C Vol. 113, pp. 13545-13550

Kapitonov, A.M.; Stupak, A.P.; Gaponenko, S.V.; Petrov, E.P.; Rogach, A.L.; Eychmuller, A. (1999). Luminescence Properties of Thiol-Stabilized CdTe Nanocrystals, cI Phys. Chem. B, Vol.103, pp. 10109-10113.

Krustok, J.; Raudoja, J.; Schon, J.H.; Yakushev, M.; Collan, H. (2000). The role of deep donordeep acceptor complexes in CIS-related compounds, Thin Solid Films, Vol. 361-362, pp. $406-410$

Kudera, S.; Carbone, L.; Manna, L. \& Parak, W. J., (2008). Growth mechanism, shape and composition control of semiconductor nanocrystals, In: Semiconductor Nanocrystal 
Quantum Dots, Rogash, A.(Eds.), pp. 1-34, Springer Verlag, ISBN 978-3-211-75235-7, Wien

Lange, H.; Artemyev, M.; Woggon, U. \& Thomsen, C. (2009). Geometry dependence of the phonon modes in CdSe nanorods, Nanotechnology Vol. 20, pp. 045705-045709

Li, J \& Wang, L. W. (2003). High Energy Excitations in CdSe Quantum Rods, Nano Letters Vol. 3, pp. 101-105

Li, J. J.; Wang, Y. A.; Guo, W. Z.; Keay, J. C.; Mishima, T. D.; Johnson, M. B.; Peng \& X. G. (2003). Large-Scale Synthesis of Nearly Monodisperse CdSe/CdS Core/Shell Nanocrystals Using Air-Stable Reagents via Successive Ion Layer Adsorption and Reaction, I Am. Chem. Soc., Vol. 125, pp.12567- 12575

Manna, L.; Scher, E. C. \& Alivisatos, A. P. (2000). Synthesis of Soluble and Processable Rod-, Arrow-, Teardrop-, and Tetrapod-Shaped CdSe Nanocrystals, el Am. Chem. Soc. Vol. 122, pp.12700- 12706

Markovich, G.; Collier, C.; Henrichs, S.; Remacle, F.; Levine, R. \& Heath, J. R. (1999) Architectonic Quantum Dot Solids, Acc. Chem. Res. Vol. 32, pp. 415-423

Murray, C. B.; Norris, D. J. \& Bawendi, M. G. (1993). Synthesis and characterization of nearly monodisperse $\mathrm{CdE}(\mathrm{E}=$ sulfur, selenium, tellurium) semiconductor nanocrystallites J Am. Chem. Soc. Vol. 115, pp. 8706- 8715

Ryu, E.; Kim, S.; Jang, E.; Jun, S.; Jang, H.; Kim, B. \& Kim, S.-W. (2009). Step-Wise Synthesis of InP/ZnS Core-Shell Quantum Dots and the Role of Zinc Acetate, Chem. Mater. Vol. 21, pp.573- 575

Saunders, A. E.; Ghezelbash, A.; Sood, P. \& Korgel, B. A. (2008). Synthesis of High Aspect Ratio Quantum-Size CdS Nanorods and Their Surface-Dependent Photoluminescence, Langmuir Vol. 24, pp. 9043-9049

Schlegel, G.; Bohnenberger, J.; Potapova, I. \& Mews, A. (2002). Fluorescence Decay Time of Single Semiconductor Nanocrystals, Phys. Rev. Lett. Vol.88, 137401.

Uehara, M.; Watanabe K.; Tajiri Y.; Nakamura, H. \& Maeda, H. (2008). Synthesis of CuInS 2 fluorescent nanocrystals and enhancement of fluorescence by controlling crystal defect, I Chem. Phys., Vol. 129, 134709

Wang, X.; Qu, L.; Zhang, J.; Peng, X.; Xiao, M. (2003). Surface-Related Emission in Highly Luminescent CdSe Quantum Dots, Nano Letters, Vol.3 , pp.1103-1106

Xie, R.; Kolb, U.; Li, J.; Basche, T. \& Mews, A. (2005). Synthesis and Characterization of Highly Luminescent CdSe-Core CdS $/ Z n_{0.5} \mathrm{Cd}_{0.5} \mathrm{~S} / \mathrm{ZnS}$ Multishell Nanocrystals, $\mathcal{C}$ Am. Chem. Soc. Vol. 127, pp. 7480-7488

Xie, R.; Rutherford, M. \& Peng, X. (2009). Formation of high-quality I-III-VI semiconductor nanocrystals by tuning relative reactivity of cationic precursors Chem. Mater. , Vol. 22, pp. 5691-5697

Xu, S.; Ziegler, J. \& Nann, T. (2008). Rapid synthesis of highly luminescent InP and InP/ZnS nanocrystals, el Mater. Chem. Vol. 18, pp.2653-2656.

Zhang, J. \& Yu, W. W. (2006). Formation of CdTe nanostructures with dot, rod, and tetrapod shapes, Appl. Phys. Lett., Vol. 89, pp. 123108-123111

Zhong, H.; Zhou, Y.; Mingfu, Y.; He, Y.; Ye, J.; He, C.; Yang, C. \& Li, Y. (2008). Controlled Synthesis and Optical Properties of Colloidal Ternary Chalcogenide CuInS 2 Nanocrystals, Chem. Mater., Vol. 20, pp.6434-6443. 
Zenkevich, E.; Cichos, F.; Shulga, A.; Petrov, E.P.; Blaudek, T. \& von Borczyskowski, C. (2005). Nanoassemblies Designed from Semiconductor Quantum Dots and Molecular Arrays, el Phys. Chem. B 109, pp. 8679-8685. 


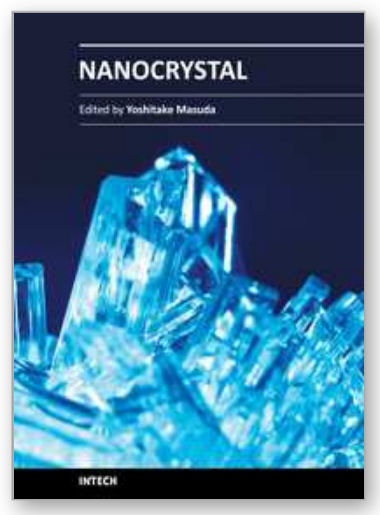

\author{
Nanocrystal \\ Edited by Dr. Yoshitake Masuda
}

ISBN 978-953-307-199-2

Hard cover, 494 pages

Publisher InTech

Published online 28, June, 2011

Published in print edition June, 2011

We focused on cutting-edge science and technology of Nanocrystals in this book. â€œNanocrystalâ€ is expected to lead to the creation of new materials with revolutionary properties and functions. It will open up fresh possibilities for the solution to the environmental problems and energy problems. We wish that this book contributes to bequeath a beautiful environment and valuable resources to subsequent generations.

\title{
How to reference
}

In order to correctly reference this scholarly work, feel free to copy and paste the following:

Young-Kuk Kim and Chul-Jin Choi (2011). Tailoring the Morphology and the Optical Properties of Semiconductor Nanocrystals by Alloying, Nanocrystal, Dr. Yoshitake Masuda (Ed.), ISBN: 978-953-307-199-2, InTech, Available from: http://www.intechopen.com/books/nanocrystal/tailoring-the-morphology-and-theoptical-properties-of-semiconductor-nanocrystals-by-alloying

\section{INTECH}

open science | open minds

\author{
InTech Europe \\ University Campus STeP Ri \\ Slavka Krautzeka 83/A \\ 51000 Rijeka, Croatia \\ Phone: +385 (51) 770447 \\ Fax: +385 (51) 686166 \\ www.intechopen.com
}

\author{
InTech China \\ Unit 405, Office Block, Hotel Equatorial Shanghai \\ No.65, Yan An Road (West), Shanghai, 200040, China \\ 中国上海市延安西路65号上海国际贵都大饭店办公楼405单元 \\ Phone: +86-21-62489820 \\ Fax: +86-21-62489821
}


(C) 2011 The Author(s). Licensee IntechOpen. This chapter is distributed under the terms of the Creative Commons Attribution-NonCommercialShareAlike-3.0 License, which permits use, distribution and reproduction for non-commercial purposes, provided the original is properly cited and derivative works building on this content are distributed under the same license. 